\title{
A comprehensive assessment of resting state networks: bidirectional modification of functional integrity in cerebro-cerebellar networks in dementia
}

\section{Gloria Castellazzi ${ }^{1,2 *}$, Fulvia Palesi $i^{2,3}$, Stefano Casali ${ }^{2,4}$, Paolo Vitali ${ }^{5}$, Elena Sinforiani ${ }^{6}$, Claudia A. M. Wheeler-Kingshott ${ }^{7 *}$ and Egidio D'Angelo ${ }^{2,4 *}$}

\author{
1 Department of Industrial and Information Engineering, University of Pavia, Pavia, Italy \\ ${ }^{2}$ Brain Connectivity Center, C. Mondino National Neurological Institute, Pavia, Italy \\ ${ }^{3}$ Department of Physics, University of Pavia, Pavia, Italy \\ ${ }^{4}$ Department of Brain and Behavioral Sciences, University of Pavia, Pavia, Italy \\ ${ }^{5}$ Brain MRI 3T Mondino Research Center, C. Mondino National Neurological Institute, Pavia, Italy \\ ${ }^{6}$ Neurology Unit, C. Mondino National Neurological Institute, Pavia, Italy \\ ${ }^{7}$ NMR Research Unit, Department of Neuroinflammation, Queen Square MS Centre, UCL Institute of Neurology, London, UK
}

\section{Edited by:}

Alexander LLoyd MacKay, University

of British Columbia, Canada

\section{Reviewed by:}

Fahmeed Hyder, Yale University, USA

Sheng Zhang, Yale University, USA

Daniela Perani, San Raffaele

University, Italy

\section{*Correspondence:}

Gloria Castellazzi, Department of

Industrial and Information

Engineering, University of Pavia, Via

Ferrata 1, I-27100 Pavia, Italy

e-mail: gloria.castellazzi@unipv.it;

Egidio D'Angelo, Department of

Brain and Behavioral Sciences,

University of Pavia, Via Forlanini 6,

1-27100 Pavia, Italy

e-mail: dangelo@unipv.it;

Claudia A. M. Wheeler-Kingshott,

NMR Research Unit, Department of

Neuroinflammation, Queen Square

MS Centre, UCL Institute of

Neurology, Queen Square, WC1N

3BG London, UK

e-mail:c.wheeler-kingshott@

ucl.ac.uk
In resting state $f M R I$ (rs-fMRI), only functional connectivity $(F C)$ reductions in the default mode network (DMN) are normally reported as a biomarker for Alzheimer's disease (AD). In this investigation we have developed a comprehensive strategy to characterize the FC changes occurring in multiple networks and applied it in a pilot study of subjects with $\mathrm{AD}$ and Mild Cognitive Impairment (MCl), compared to healthy controls $(\mathrm{HC})$. Resting state networks (RSNs) were studied in $14 \mathrm{AD}$ ( $70 \pm 6$ years), $12 \mathrm{MCl}$ ( $74 \pm 6$ years), and $16 \mathrm{HC}$ (69 \pm 5 years). RSN alterations were present in almost all the 15 recognized RSNs; overall, 474 voxels presented a reduced $\mathrm{FC}$ in $\mathrm{MCl}$ and 1244 in $\mathrm{AD}$ while 1627 voxels showed an increased $\mathrm{FC}$ in $\mathrm{MCl}$ and 1711 in $\mathrm{AD}$. The RSNs were then ranked according to the magnitude and extension of FC changes $(g F C)$, putting in evidence 6 RSNs with prominent changes: DMN, frontal cortical network (FCN), lateral visual network $(L V N)$, basal ganglia network (BGN), cerebellar network (CBLN), and the anterior insula network (AIN). Nodes, or hubs, showing alterations common to more than one RSN were mostly localized within the prefrontal cortex and the mesial-temporal cortex. The cerebellum showed a unique behavior where voxels of decreased $g F C$ were only found in $A D$ while a significant $g F C$ increase was only found in $\mathrm{MCl}$. The $g F C$ alterations showed strong correlations $(p<0.001)$ with psychological scores, in particular Mini-Mental State Examination (MMSE) and attention/memory tasks. In conclusion, this analysis revealed that the DMN was affected by remarkable FC increases, that FC alterations extended over several RSNs, that derangement of functional relationships between multiple areas occurred already in the early stages of dementia. These results warrant future work to verify whether these represent compensatory mechanisms that exploit a pre-existing neural reserve through plasticity, which evolve in a state of lack of connectivity between different networks with the worsening of the pathology.

Keywords: Alzheimer disease, mild cognitive impairment, resting state fMRI, functional connectivity alterations, cerebro-cerebellar networks

\section{INTRODUCTION}

Alzheimer's disease $(\mathrm{AD})$ is a progressive neurodegenerative disorder characterized by diffuse cortical atrophy, most pronounced in the mesial-temporal lobe (Sluimer et al., 2009; Ferreira et al.,

Abbreviations: AD, Alzheimer disease; AIN, anterior insula network; AN, auditory network; BA, Brodmann area; BGN, basal ganglia network; CBLN, cerebellar network; LN, language network; DMN, default mode network; ECN, executive control network; HC, healthy controls; ICA, independent component analysis; LVN, lateral visual network; MCI, mild cognitive impairment; FCN, frontal cortical network; MVN, medial visual network; rs-fMRI, resting state functional magnetic resonance imaging; SMN, sensory motor network; SN, salience network; TPN, task positive network; VAN, ventral attention network.
2011). The pathological hallmarks of $\mathrm{AD}$ are the accumulation of $\beta$ amyloid $\left(\mathrm{A} \beta_{1-42}\right)$ plaques and tau tangles concentrated in the prefrontal and mesial-temporal lobe (Blennow et al., 2006; Holtzman et al., 2011; Serrano-Pozo et al., 2011; Herskovits et al., 2013). Mild cognitive impairment (MCI) is a less sever clinical state that can evolve in $\mathrm{AD}$ but demonstrate less pronounced brain atrophy. It is possible that, during the progression from $\mathrm{MCI}$ to $\mathrm{AD}$, a complex rearrangement occurs in brain networks. For example, PET-MRI studies have shown that, in $\mathrm{MCI}$, hypometabolism is predominant in precuneus/posterior cingulum, (Morbelli et al., 2010), while amyloid deposition is 
more conspicuous in prefrontal lobe (La Joie et al., 2012). These findings suggest a dissociation between hystopathological and functional changes, which can extend over brain regions not affected by neurodegeneration and may differ in nature and localization at different stages of the pathology.

Functional magnetic resonance imaging (fMRI) is a leading candidate for assessing changes in functional connectivity (FC), suitable to study MCI and AD thanks to the short acquisition protocol without the need of performing a task. By measuring FC between spatially distinct brain regions, resting-state fMRI (rs-fMRI) allows the identification of several networks (RSNs) (Calhoun et al., 2001; Beckmann et al., 2005; Damoiseaux et al., 2006, 2008; Cole et al., 2010), in which separate brain areas show MR signal correlations in the absence of any specific external stimulation (Biswal et al., 1995; Cordes et al., 2001; Greicius et al., 2003; Fox and Raichle, 2007; Zhang and Raichle, 2010). Because of the striking overlap between areas of amyloid deposition and areas involved in the default mode network (DMN) (Mormino et al., 2011), most rs-fMRI studies in AD patients have focused the attention on alterations of the DMN (Jones et al., 2011; Petrella et al., 2011). The DMN is active during episodic and autobiographical memory retrieval and shows decreased activity during cognitive tasks demanding attention to external stimuli (Raichle et al., 2001; Greicius et al., 2003, 2004; Wu et al., 2011; Brier et al., 2012; Koch et al., 2012). In AD, the DMN has shown decreased FC in the precuneus and posterior cingulate cortex, revealing a good matching between reduced FC, cortical atrophy and memory impairment (Binnewijzend et al., 2012). Only a few studies suggested also the involvement of other RSNs, including visual cortex, basal ganglia and cerebellum (Agosta et al., 2012; Binnewijzend et al., 2012; Brier et al., 2012). Interestingly, the cerebellum is a common area that can be thought as a node of several RSNs including the DMN. Although previous works have suggested a cerebellar involvement in AD and MCI (Wang et al., 2007; Kaufmann et al., 2008; Thomann et al., 2008; Bai et al., 2009, 2011b; Solé-Padullés et al., 2009; Teipel et al., 2013), the alteration in $\mathrm{FC}$ in the cerebellum in $\mathrm{AD}$ and MCI remains unclear.

In this work we have characterized the changes occurring in multiple RSNs in two groups of patients, with clinical diagnosis of $\mathrm{MCI}$ and $\mathrm{AD}$, compared to healthy subjects. In order to take into consideration positive and negative alterations in terms of both FC amplitude and voxels extension, we introduced a generalized FC $(g F C)$ parameter. Then we ranked the networks and identified key patterns when comparing changes between MCI and $\mathrm{AD}$ and $\mathrm{HC}$ and we investigated the presence of hubs of alterations (nodes) at the intersection of multiple networks. We finally assessed clinical correlations and characteristics that could be useful in future longitudinal studies aimed at addressing the issue of predicting clinical conversion.

\section{METHODS}

\section{SUBJECTS}

A total of 56 subjects (mean age $70 \pm 6$ ) were recruited for this study among those suffering from subjective or objective memory complaint, attending regularly the Memory Clinic of the Neurological Institute C. Mondino, Pavia, Italy. Subjects were selected based on their neuropsychological examination using a standardized battery of tests (see section below), which evaluated different cognitive domains (Spinnler and Dall'ora, 1987). Exclusion criteria were: age $>80$ years, significant medical, neurological (different from $\mathrm{AD}$ ) psychiatric disease as well as significant cerebrovascular disease (Hachinski et al., 1975; Binnewijzend et al., 2012). In detail, patients with significant Central Nervous System (CNS) disorders other than AD (e.g., Parkinson's disease and other extra-pyramidal disorders, multiple sclerosis, epilepsy, significant focal or vascular intracranial pathology, clinical evidence of cerebrovascular accident, and/or previous head injury with loss of consciousness) were excluded. Written informed consent was provided by all the subjects or their lawful caregiver. Fourteen patients were excluded because of data quality, mainly motion. Some images had such artifacts that were excluded on visual inspection. When in doubt, realignment parameters were also considered and data excluded if the subject had a maximum displacement in any of the cardinal directions $(x, y, z)$ that was larger than $3 \mathrm{~mm}$, or a maximum spin $(\mathrm{x}, \mathrm{y}, \mathrm{z})$ larger than $3^{\circ}$. After inspection, 42 subjects of the original 56 were included in this study. Based on the clinical evaluation, subjects were divided into two groups: 14 patients ( 8 females, mean age $74 \pm 6)$ were classified as AD (NINCDS2-ARDAcriteria) (McKhann et al., 2011) and 12 patients ( 10 females, mean age $70 \pm 6$ ) as MCI (Petersen et al., 2001, 2009; Petersen, 2009). MCI subjects were classified as having amnestic MCI (a-MCI) if the memory was the only impaired domain, multi-domain MCI (md-MCI) if the impairment was not limited to the memory. Based on this rule, 3 of the $12 \mathrm{MCI}$ subjects recruited for this study were classified as a-MCI, while the remaining 9 patients were md-MCI. Because of the small sample of a-MCI, all the MCI subjects (both a-MCI and md-MCI) were combined in a unique group. In order to obtain a reference metric for our findings, $16 \mathrm{HC}$ (10 females, mean age $69 \pm 5$ ) were recruited on a volunteer base through a local recreational association ("Argento Vivo," Bereguardo, PV) and underwent the MRI examination.

\section{CLINICAL AND NEUROPSYCHOLOGICAL EXAMINATION}

Subjects underwent clinical and neuropsychological testing, which evaluated different cognitive domains (Spinnler and Dall'ora, 1987; Spinnler and Tognoni, 1987) including: MiniMental State Examination (MMSE) (Folstein et al., 1975), trial making test part A and B (TMT-A and TMT-B) (Reitan, 1958), memory for prose (MP) (Novelli et al., 1986), category fluency (CF), and semantic fluency (SF) (Randolph et al., 1993), Rey complex figure copy test (ROCF-copy) and Rey complex figure recall (ROCF-rec) (Osterrieth, 1944; Caffarra et al., 2002). For each test age-, gender-, and education-corrected scores were calculated from the raw scores. Corrected scores were then transformed in equivalent scores ranging from 0 (pathological) to 1 (lower limit of normal) and 2-4 (normal). Only corrected scores were used in the statistical analysis.

\section{MRI ACOUISITIONS}

All data were acquired using a 1.5T MR Philips Intera Gyroscan (Philips Healthcare, Best, The Netherlands) with an 8-channel head (SENSE) third-party coil. For each subject a fast field 
echo-planar imaging (FFE-EPI) protocol was acquired for rs-fMRI with $T R / T E=3000 / 60 \mathrm{~ms}$, voxel size $=2.2 \times 2.2 \times$ $4 \mathrm{~mm}^{3}, \mathrm{FOV}=250 \times 250 \mathrm{~mm}^{2}, 26$ slices, SENSE factor $=3.1$, 100 repeated volumes. For anatomical reference a volumetric 3DT1-weighted acquisition was also collected using a fast field echo $(\mathrm{FFE})$ sequence $\left(T R / T E=8.6 / 4 \mathrm{~ms}\right.$; flip angle $8^{\circ}$; 170 sagittal slices; slice thickness $=1.2 \mathrm{~mm}$; FOV $=240 \mathrm{~mm}$; acquisition matrix $=192 \times 192$, reconstructed to $256 \times 256$; in-plane resolution $1.25 \times 1.25 \mathrm{~mm}^{2}$, reconstructed to $0.94 \times 0.94 \mathrm{~mm}^{2}$ ).

All the MRI analysis was performed on a workstation with Linux Ubuntu 12.04, running SPM8 (Wellcome Department of Cognitive Neurology, http://www.fil.ion.ucl.ac.uk/), Matlab R2009a (The MathWorks, Natick, Mass, USA http://www. mathworks.com/) and FSL (FMRIB Software Library, version 4.1.9, http://www.fmrib.ox.ac.uk/fsl/).

\section{fMRI ANALYSIS}

For each recruited subject rs-fMRI images were analyzed using Independent Component Analysis (ICA) to characterize RSNs. ICA results were analyzed using the Multivariate Exploratory Linear Optimized Decomposition into Independent Components (MELODIC) method as implemented in FSL.(Beckmann et al., 2005)

\section{Data pre-processing}

Individual subject's pre-processing consisted in motion correction, brain extraction, spatial smoothing using a Gaussian kernel of full-width-at-half-maximum (FWHM) of $6 \mathrm{~mm}$, and high pass temporal filtering equivalent to $150 \mathrm{~s}(0.007 \mathrm{~Hz})$. rs-fMRI volumes were then registered to the individual's structural 3DT1 scan using FMRIB's Linear Image Registration Tool (FLIRT) and subsequently to standard space (MNI152) using FMRIB's Nonlinear Image Registration Tool (FNIRT) with default options.

\section{Independent component analysis (ICA)_identification of RSNs}

Pre-processed functional data, containing 100 time points (volumes) for each subject, were temporally concatenated across subjects to create a single 4-dimensional data set. The dataset was decomposed into independent components (ICs), with an automatic estimation for the number of components, which resulted in spatial maps, each with an associated time course. Model order was estimated using the Laplace approximation to the Bayesian evidence for a probabilistic principal component model. Some of the ICs were identified as noise while others as RSNs, based on previous literature (Beckmann et al., 2005; Damoiseaux et al., 2006; Smith et al., 2009; Cole et al., 2010). This method is run on the entire dataset (i.e., the selected 42 subjects) and decomposes data into spatial maps that are the ICs relative to the total processed dataset, or the multi-subject ICA components. This means that ICs are the same for each subject and represent the maps within which inference between groups ( $\mathrm{AD}, \mathrm{MCI}$, and $\mathrm{HC}$ ) is then evaluated applying dual regression.

\section{Dual regression-evaluation of group differences within the RSNs}

A non-parametric permutation test, referred to as "dual regression" technique, was then applied to compare group-specific FC maps for each independent spatial component. In particular with this analysis differences between $\mathrm{HC}, \mathrm{MCI}$, and $\mathrm{AD}$ groups were tested using 4 different comparisons, which we will refer to as contrasts (MCI < HC; $\mathrm{AD}<\mathrm{HC}$; MCI $>\mathrm{HC}$; and $\mathrm{AD}>\mathrm{HC}$ ). Dual regression allows for between-subjects analysis by carrying out a voxel-wise comparisons of the resting FC (Filippini et al., 2009). In this study the dual regression analysis was carried out on the total ICs using age, gender and education level as additional covariates in the permutation tests (http://fsl.fmrib.ox.ac. $\mathrm{uk} / \mathrm{fsl} /$ fslwiki/DualRegression).

In detail, spatial ICs were used in a linear model fit against each individual rs-fMRI data set (spatial regression), to create matrices that described the temporal dynamics for each component and subject separately. These matrices were used in a linear model fit against the associated subject's rs-fMRI data set (temporal regression), to estimate subject-specific spatial correlation maps. Subsequently spatial maps of all subjects were collected into single 4-dimensional files for each original independent component and tested voxel-wise for statistically significant differences between groups using nonparametric permutation tests $(10,000$ permutations) (Filippini et al., 2009; Binnewijzend et al., 2012). The resulting statistical maps were family-wise error (FWE) corrected for multiple comparisons using the threshold-free cluster enhancement (TFCE) method. In detail, TFCE uses the raw statistic maps produced in the initial steps of the dual regression methods and yields images, in which the voxel-wise values represent the amount of cluster-like local spatial support (Smith and Nichols, 2009). Voxels that survived a statistical threshold of $p \leq 0.05$ were considered significant (Binnewijzend et al., 2012) and were saved as tstat ${ }_{\mathrm{FC}}$ maps.

\section{Global network analysis-ranking of RSN alterations}

In order to study FC changes within each RSN and to establish a ranking of the networks in terms of their alterations, for each contrast we defined a global parameter taking into account (1) the extension of the clusters and (2) the magnitude of the FC changes. For each RSN, we considered (1) the number of altered voxels in the tstat ${ }_{\mathrm{FC}}$ map $\left(N_{\text {tstatFC }}\right)$ with respect to the total number of voxels of the RSN itself $\left(N_{R S N}\right)$. For each RSN we calculated (2) the overall FC change of the group (meanFC) by firstly averaging each subject's tstat ${ }_{\mathrm{FC}}$ map and then evaluating its mean for each group to give meanFC $C_{H C}$, for the $\mathrm{HC}$, and meanFC $C_{P}$, for patients, with $\mathrm{p}=\mathrm{MCI}$ or $\mathrm{AD}$. We then combined these parameters in a new index, called global FC ( $g F C)$ index as follows:

$$
g F C=\left|\left(\frac{\text { meanFC }_{H C}-\text { meanFC }_{P}}{m e a n F C_{H C}}\right)\right| \cdot \frac{N_{\text {tstatFC }}}{N_{R S N}}
$$

For each contrast, we were therefore able to rank the RSN alterations in terms of their decreasing $g F C$ values.

\section{Individual network analysis—regional quantification of RSN alterations}

To assess spatial distributions of RSNs changes we divided the brain according to cortical regions (Brodmann, 2006; Diedrichsen et al., 2009) into seven different areas, (l): cerebellum (C), prefrontal (PreF), precentral (PreC), limbic (L), occipital $(\mathrm{O})$, parietal $(\mathrm{P})$, and temporal $(\mathrm{T})$. In particular, PreF includes the inferior, middle and superior frontal cortices. Each of the 
Table 1 | Brain subdivision used to assess the spatial distributions of RSN changes.

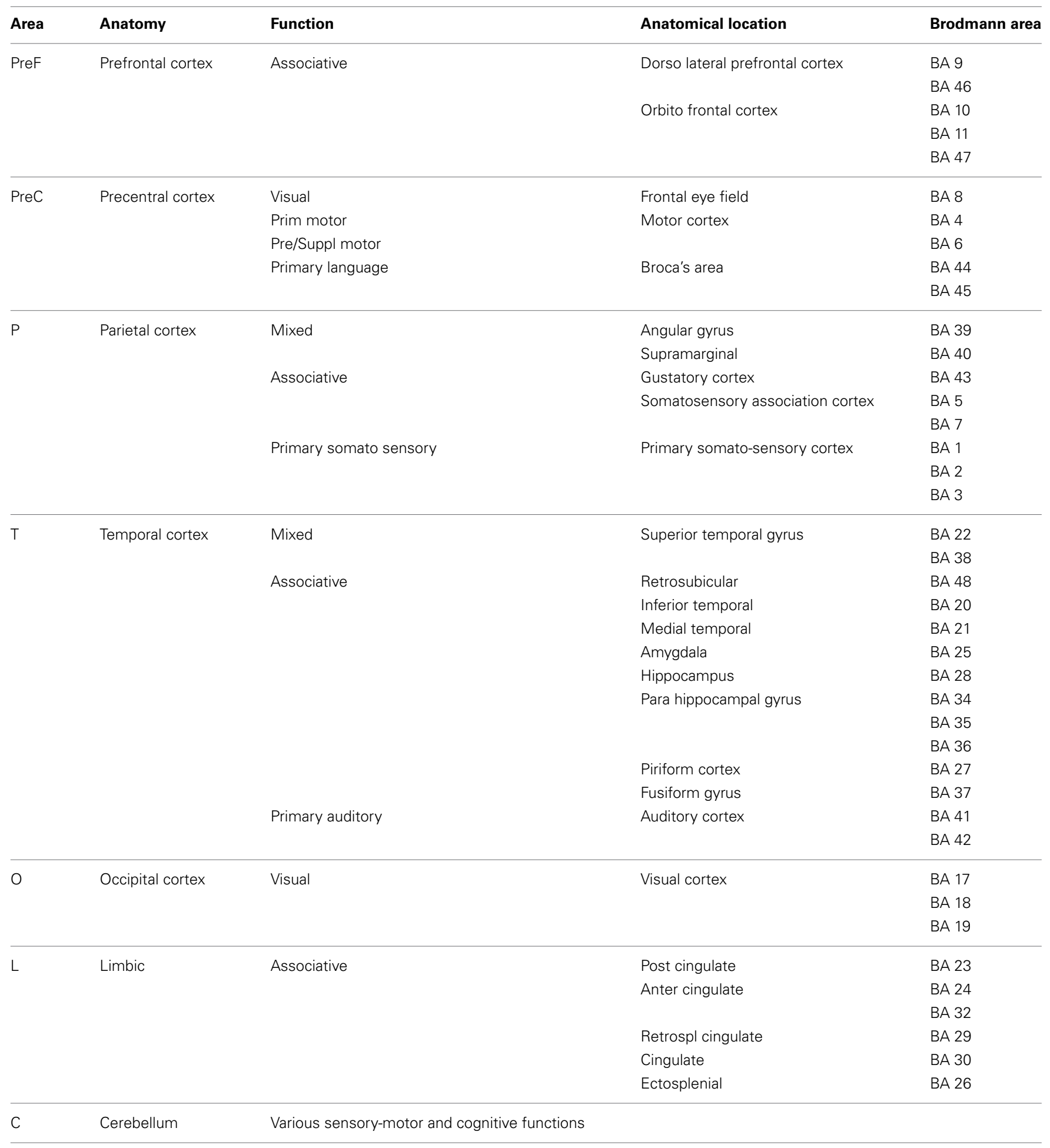

seven anatomical regions has been obtained grouping different Brodmann areas on anatomical and functional basis (see Table 1 for details). The temporal ( $\mathrm{T}$ ) sub-area includes the lateral (inferior, middle, and superior temporal gyri), inferior (fusiform gyrus), and mesial (amygdala, parahippocampal gyrus, and hippocampus) temporal cortices. Similarly to the evaluation of the gFC index, for each contrast we considered both the FC changes and the extension of such changes in each areas $l$. We used the tstats $_{\mathrm{FC}}$ maps to quantify the mean value of altered $F C$ within each RSN within each sub-area $[$ meanFC(l)], and calculated the total number of altered voxels $\left[N_{\text {tstatFC }}(1)\right]$ for the same RSN. We defined relative changes compared to the overall mean $F C(1)_{H C}$ in 
$H C$ and the total number of voxels in the considered RSN, $N_{R S N}$. For each sub-area, $l$, and for each $R S N$ we then combined these parameters into a regional global index, $\mathrm{rgFC}(\mathrm{l})$ as follows:

$$
\operatorname{rgFC}(l)=\left|\left(\frac{\text { meanFC }(l)_{H C}-\text { meanFC }(l)_{P}}{\operatorname{meanFC}(l)_{H C}}\right)\right| \cdot \frac{N_{t s t a t F C}(l)}{N_{R S N}}
$$

\section{Definition of nodes}

On the basis of the previous mentioned parcellation of the brain into seven different sub-regions ( $\mathrm{C}$, PreF, PreC, L, O, P, and T), we considered as nodes or hubs those sub-regions in which multiple networks presented coexistent functional alteration. For each node we were able to calculate the percentage of functional alteration calculating the ratio between the total number of altered voxels within the node and the total number of voxels that fall at least within a RSN.

\section{VOXEL BASED MORPHOMETRY (VBM) ANALYSIS}

To account for possible interactions between structural differences as measured by atrophy and the observed FC differences between $\mathrm{AD}, \mathrm{MCI}$, and $\mathrm{HC}$, we performed Voxel Based Morphometry (VBM) analysis on the 3DT1-weighted images, using SPM8 following a standard VBM protocol (Testa et al., 2004; Boccardi et al., 2005; Frisoni et al., 2005; Mechelli et al., 2005; Pennanen et al., 2005; Filippini et al., 2006). VBM analysis was performed on all 42 subjects included in the present study [ 14 AD patients, $12 \mathrm{MCI}$, and 16 healthy controls (HC)].

Briefly, probability maps were computed for gray matter (GM), white matter (WM), and cerebro-spinal fluid (CSF) (Good et al., 2002). Following these steps: for each subject 3D T1-weighted images were normalized to a high resolution T1 MNI152 template through affine and nonlinear transformations. The resulted image was then segmented into GM, WM, and CSF using the customized priors, masked to remove non-brain tissue voxels, modulated, and finally smoothed with a $8 \mathrm{~mm}$ Gaussian kernel (Ashburner and Friston, 2001).

Statistical analysis was performed on each tissue priors using the general linear model (GLM) framework and the resulting t-statistic maps were thresholded at $p<0.001$. In order to reduce the number of false positives we set an extent threshold of 30 voxels (Ashburner and Friston, 2001; Mechelli et al., 2005).

In order to verify to what extent FC changes are co-localized with VBM changes, in particular within the DMN we: (1) calculated the overlap between voxels in the DMN with those showing the GM atrophy; (2) we calculated the overlap between the FC decrease in the DMN and voxels affected by GM atrophy.

\section{EFFECTS OF CORTICAL ATROPHY ON FC CHANGES}

As a sensitivity analysis, to verify whether cortical volume influenced the fMRI results and in particular the FC changes, a second dual-regression analysis was carried out on all the ICs using the relative GM volume, expressed as ratio between GM absolute volume $\left(\mathrm{mm}^{3}\right)$ and intracranial volume $\left(\mathrm{mm}^{3}\right)$ as a further additional covariate in the permutation tests (Oakes et al., 2007; Damoiseaux et al., 2008; Binnewijzend et al., 2012).

\section{NON-IMAGING STATISTICS}

All other statistical analyses were performed using SPSS (version 17.0; SPSS, Chicago, IL, USA). For continuous measures, differences between groups were assessed using one-way analysis of variance (ANOVA) with post-hoc Tamhane tests to correct for multiple comparisons. Data of TMT-A, ROCF-copy, and ROCFrec were log transformed as Kolmogorov-Smirnov tests showed they were not normally distributed. We performed a $\chi^{2}$ test to compare frequency distributions of age, gender and education level. We performed Pearson's correlation analysis to assess correlations between regional FC values ( $\mathrm{rgFC}$ index) within the RSNs (extracted mean $z$-values from the clusters of voxels showing regional FC differences) and neuropsychological test results. In order to facilitate the understanding of all the statistical comparisons between $\mathrm{r} g F C$ and neuropsychological scores we performed the correlation analysis considering the inverse of TMT-A (1/TMT-A) and of the TMT-B (1/TMT-B) so that we obtained the same trend, i.e., the lower the score the more severe the impairment. A very strict statistical threshold of $p<0.001$ was considered for studying the correlation between the $\mathrm{r} g F C$ index and neuropsychological data.

\section{RESULTS}

In this rs-fMRI investigation, 15 RSNs were identified. RSN alterations in $\mathrm{MCI}$ and $\mathrm{AD}$ compared to $\mathrm{HC}$ were observed in several networks and were ranked taking into account FC changes (both increased and decreased) as well as voxel extension changes, according to the $g F C$ parameter (see Methods). This strategy put into evidence 6 RSNs that were prominently involved with different patterns in groups of subjects with different stages of dementia. For each of these 6 RSNs we also extensively analyzed FC changes based on the $\mathrm{r} g F C$ index to assess the regional involvement of different cortical areas. A comprehensive analysis of these changes is presented in the sections below. All the changes reported in this study were significant at $p<0.05$, TFCE-corrected.

\section{COMPARISON OF SUBJECT'S POPULATIONS}

No differences in age, sex or level of education distinguished the $\mathrm{AD}, \mathrm{MCI}$ and $\mathrm{HC}$ groups. However, as expected from the pathological state of MCI and $\mathrm{AD}$, differences in neuropsychological tests were observed between themselves and $\mathrm{HC}$ (Table 2). Multiple ANOVA revealed significantly different scores between the groups $(p<0.001)$ for the MMSE examining cognitive impairment, the TMT examining visual attention and task switching, the MP examining episodic memory, the RPCF-rec examining visuospatial construction, visuographic memory as well as planning and executive functions on recall. However, no differences in ROCF-copy index, were observed ( $p=0.133)$. By considering the specific ANOVA subgroups, AD were worse than either MCI or HC in all neuropsychological tests on cognitive performance (MMSE, TMT, MP, ROCF-rec), while MCI were worse than $\mathrm{HC}$ only in a limited set of tests on memory and attention (MP and TMT).

\section{RESTING STATE NETWORKS IDENTIFICATION}

All subjects were scanned for rs-fMRI and the data were analyzed with ICA (see Methods for details). This processing resulted in 41 
Table 2 | Demographics and neuropsychological ANOVA test between groups.

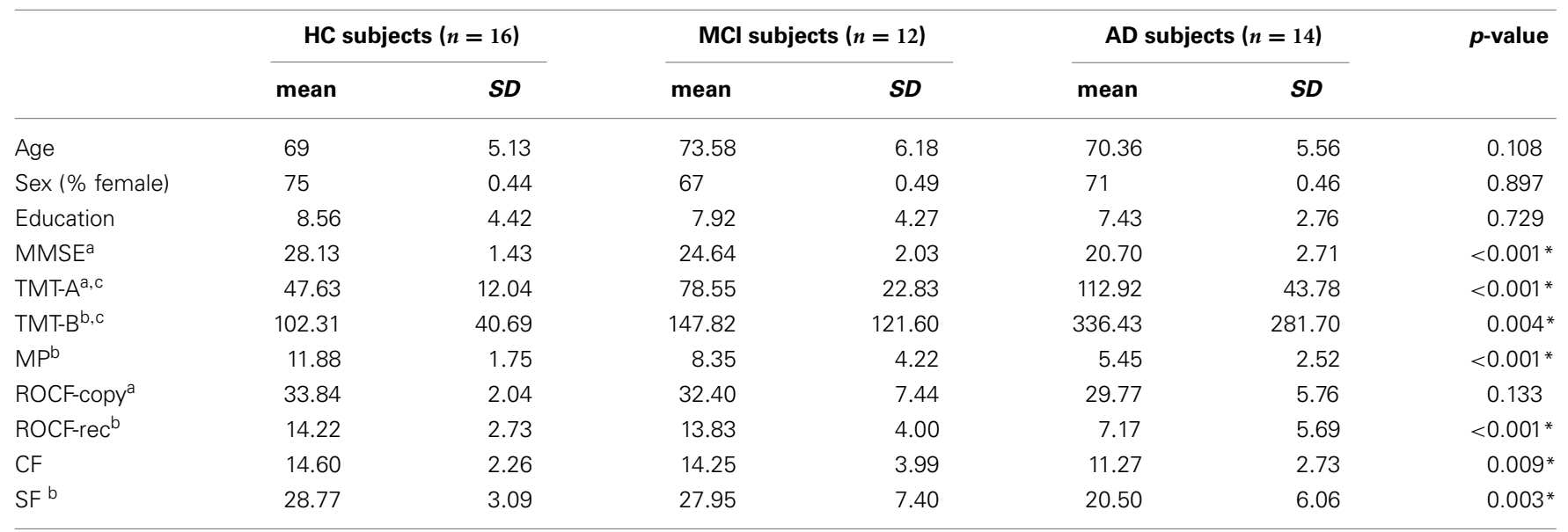

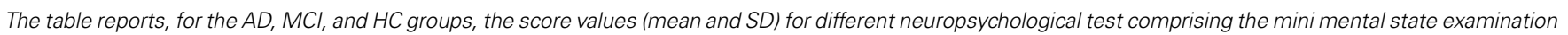

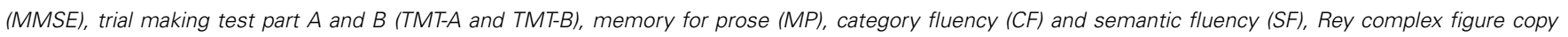
test (ROCF-copy) and Rey complex figure recall (ROCF-rec). p-values show statistically significant differences between the $\mathrm{HC}$, $\mathrm{MCl}$ and $A D$ groups. ${ }^{*} p<0.01$ threshold for statistically significant difference between groups. ${ }^{a}$ Missing data of 1-2 subjects. ${ }^{b}$ Missing data of 3-10 subjects. ${ }^{c}$ Lower scores indicate better (faster) performance.

independent components, 23 of which were recognized as part of 15 full RSNs based on their frequency spectra and spatial patterns (Beckmann et al., 2005; Damoiseaux et al., 2006; Smith et al., 2009; Cole et al., 2010). This means that, out of the 23 ICs that were not cataloged as noise, 8 ICs were not full RSNs, but portions of the remaining 15 full RSNs whose frequency patterns was altered by noise and were therefore discarded. The remaining 18 components, that made up the 41 found by ICA, reflected artifacts like movement, physiological noise or CSF partial volume effect (Gour et al., 2014).

The 15 RSNs were (Figure 1): auditory network $(A N)$, sensory motor network $(S M N)$, medial visual network $(M V N)$, lateral visual network $(L V N)$, task positive network (TPN), executive control network $(E C N)$, right and left ventral attention networks $(V A N)$, default mode network $(D M N)$, salience network $(S N)$, cerebellar network $(C B L N)$, basal ganglia network $(B G N)$, frontal cortical network $(F C N)$, anterior insula network (AIN), language network $(L N)$.

The $A N$ is responsible for auditory processing and is located in the bilateral superior temporal gyrus, involving the primary and secondary auditory cortices. The $S M N$ is responsible for sensory-motor processing and includes the primary somatosensory, primary motor, premotor, and supplementary motor cortices as well as the cerebellum. The LVN and MVN are the networks responsible for visual processing, and are located in the lateral and medial parts of the occipital lobe, respectively, involving secondary and primary visual cortex, and also involve the cerebellum. The TPN is activated during task-oriented behaviors and includes bilateral dorsolateral and ventral prefrontal cortex, insula and primary sensory-motor areas. The ECN is responsible for executive processing, including perception, action selection, memory retrieval and emotional evaluation: it includes bilaterally the superior, middle and ventro-lateral prefrontal cortices, the anterior cingulate, and the paracingulate gyri. The VAN is the networks involved in the attentional processing: VAN (left and right) is largely lateralized in the temporal-parietal junction, in the ventral frontal cortex, in the insula and in the contralateral cerebellum. The $S N$ mediates the function of other networks and includes bilaterally the anterior cingulate cortex, premotor, supplementary motor areas, and anterior insula (Bonnelle et al., 2012). The DMN, which is active during episodic and autobiographical memory retrieval and shows decreased activity during cognitive tasks demanding attention to external stimuli (Raichle et al., 2001; Greicius et al., 2003, 2004), includes the bilateral inferior parietal cortex, the precuneus, anterior and posterior cingulate cortex, mesial-temporal structures including dorso-lateral prefrontal cortex, thalamus and cerebellum. The $B G N$ is involved in emotional processing and includes the amygdala, striatum and lateral globus pallidus, mammillary bodies, hypothalamus, and the ventral tegmental area of midbrain. The FCN is involved in core mental functions at the basis of human social behaviors (Spreng et al., 2009) and includes the bilateral anterior cingulate cortex and boundary areas between prefrontal cortex (middle and inferior frontal gyri) and orbito-frontal cortex (Janes et al., 2012). The AIN is involved in cognitive control and orienting attention (Corbetta et al., 2002) and (Cole and Schneider, 2007) includes bilaterally the areas of insula, inferior frontal gyrus, anterior cingulate, the superior temporal gyrus, and cerebellum. The CBLN network is intrinsic to cerebellum and includes bilaterally large areas centered in crus I and crus II, cerebellar tonsil, the IV, V, VI, VIII, and IX lobes, dentate, declive, vermis, culmen, uvula, tuber, pyramis, medulla, and nodule. Finally, the LN involves the bilateral post cerebellum and inferior parietal lobule, the left inferior temporal gyrus and precuneus, as well as the inferior, middle, and superior frontal giri.

$L V N, M V N, A N, T P N$, and $S M N$ are networks directly related to sensori-motor processing, while DMN, SN, FCN, ECN, AIN, $B G N, V A N$, and $L N$, are prominently associated with higher cognitive functions. The cerebellum showed components not only in the intrinsic CBLN but also in SMN, MVN, LVN, DMN, 


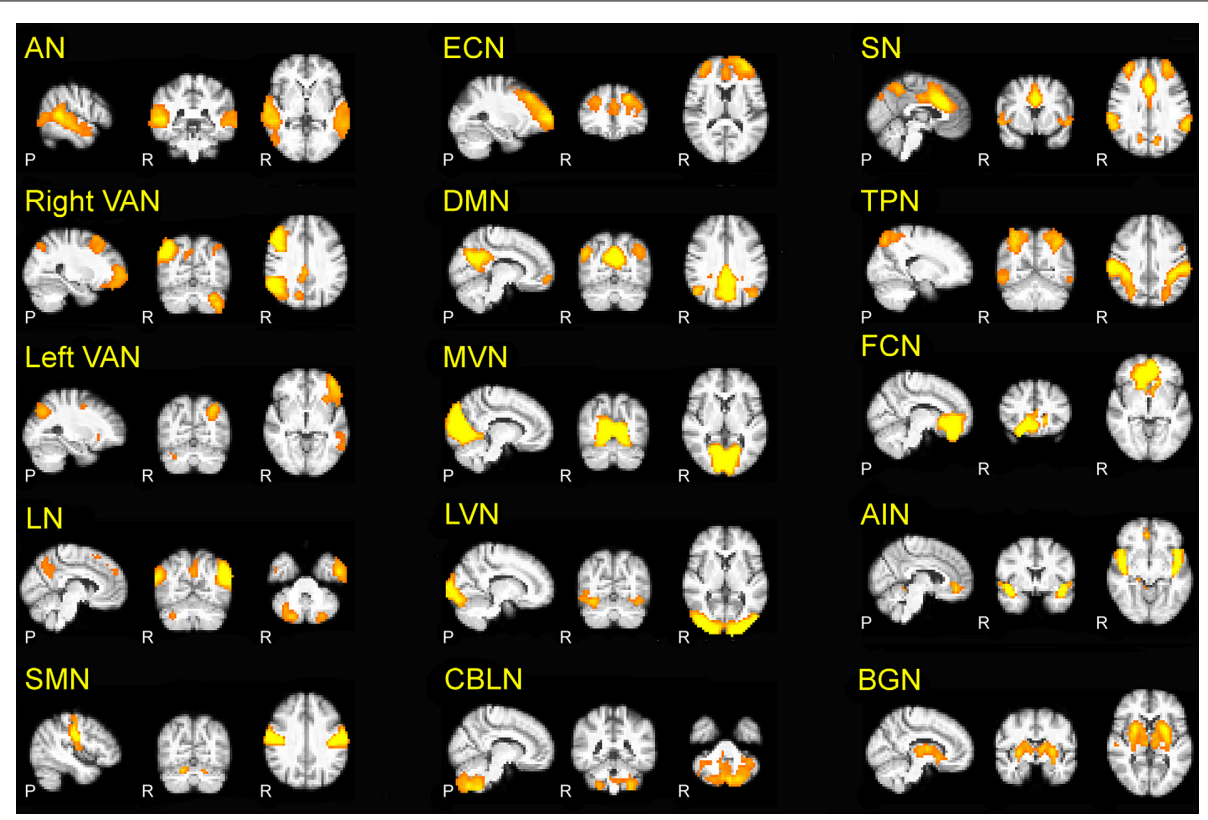

FIGURE 1 | The 15 RSNs identified in the present investigation. Auditory network (AN), right and left ventral attention networks (VAN), language network (LN), sensory-motor network (SMN), executive control network (ECN), default mode network (DMN), medial visual network (MVN), lateral visual network (LVN), cerebellar network (CBLN), salience network (SN), task positive network (TPN), basal ganglia network (BGN), frontal cortical network (FCN), anterior insula network (AIN). In this and the following figures, the $3 \mathrm{D}$ scans are projected onto three representative sections chosen on the sagittal (left), coronal (middle) and axial (right) planes.

Table 3 | FC alterations within the 15 RSNs.

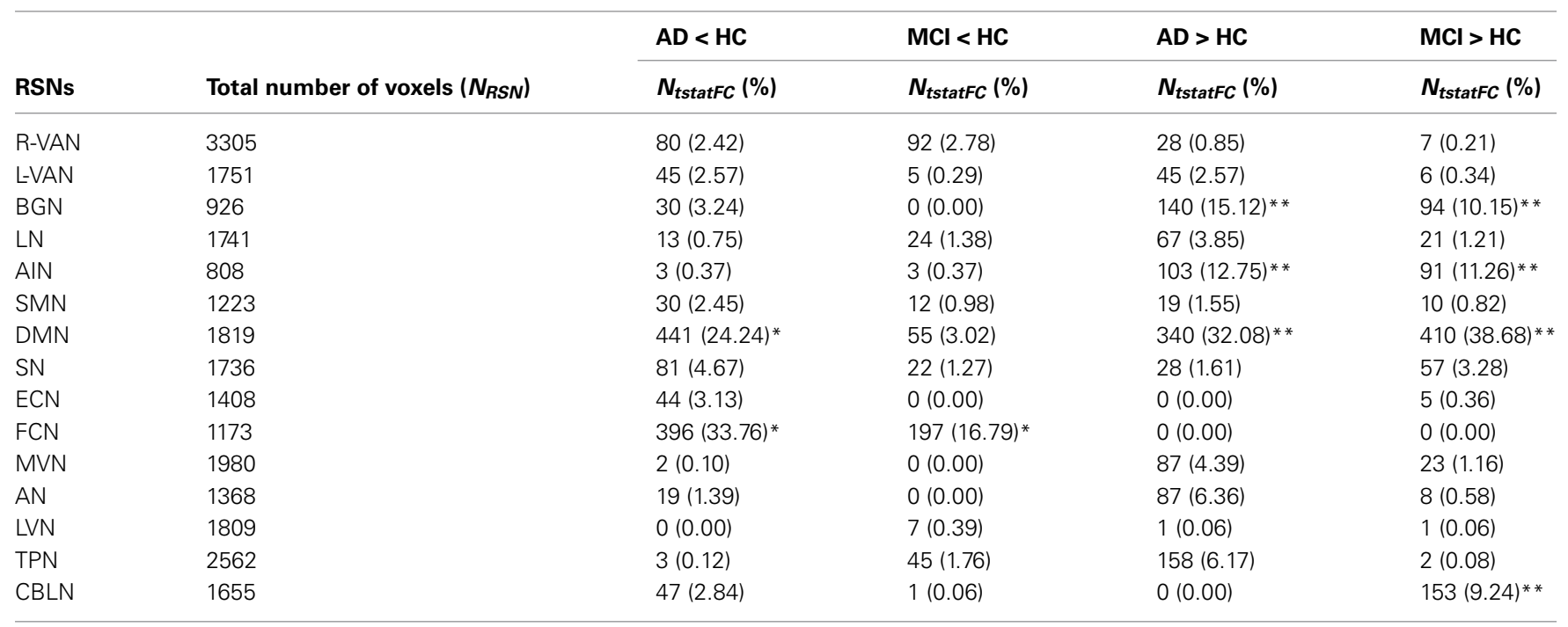

*Largest FC decreases (prefrontal areas: inferior, middle, and superior frontal giri). ${ }^{*}$ Largest FC increases (cuneus, precuneus, cerebellum).

$A I N, V A N, L N$ in line with the involvement of cerebellum both in sensory-motor and cognitive processing (Schmahmann et al., 1999; D’Angelo and Casali, 2012; Stoodley, 2012; Stoodley et al., 2012).

\section{EVALUATION OF GROUP DIFFERENCES WITHIN THE RSNs}

Our results are summarized in Table 3 and revealed, both in MCI and AD, a widespread FC alteration involving all the 15 RSNs. As shown in Figures 2, 3, depending on the RSN there was either a FC reduction or a FC increase, or both (in different sub-regions of the same RSN).

\section{Reduced FC in multiple RSNs}

Several RSNs presented a FC reduction both in MCI (for a total of 474 voxels affected) and in $\mathrm{AD}$ (for a total of 1244 voxels affected), but the areas of reduced FC were less extended in MCI than AD. 


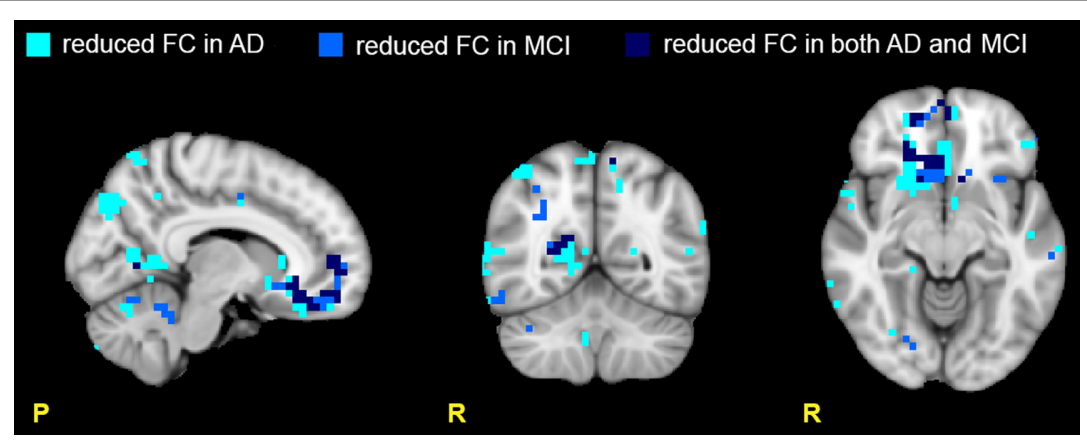

FIGURE 2 | Areas with reduced FC in the RSNs. Images show areas with reduced FC ( $p \leq 0.05$, TFCE-corrected) in AD compared to $\mathrm{HC}$ (light blue), in $\mathrm{MCl}$ compared to $\mathrm{HC}$ (blue), and in both $\mathrm{AD}$ and $\mathrm{MCl}$ compared to $\mathrm{HC}$ (dark blue). Note that the areas of reduced $\mathrm{FC}$ are mainly located in the prefrontal (PreF) and precentral (PreC) areas. Moreover ADs present a larger number of areas with reduced FC than $\mathrm{MCl}$ suggesting a more extended functional corruption in $A D$ than $\mathrm{MCl}$.

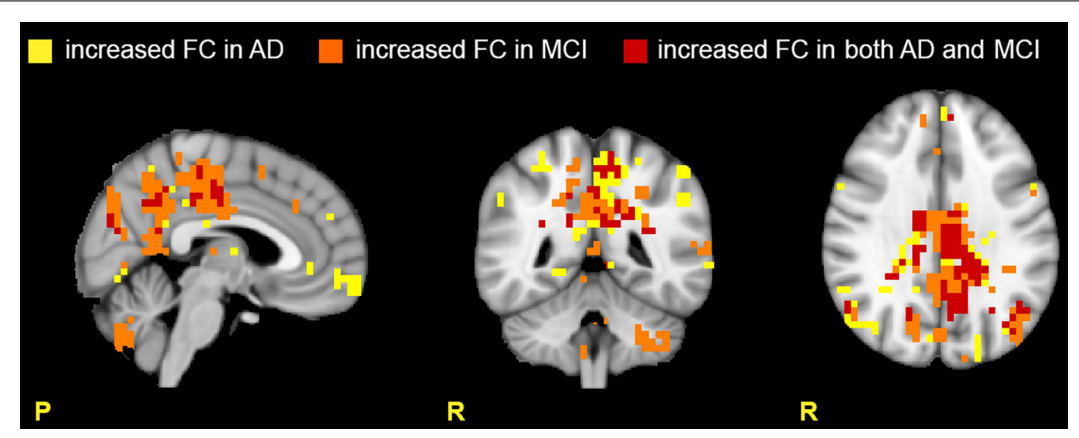

FIGURE 3 | Areas with increased FC in the RSNs. Images show areas with increased FC ( $p \leq 0.05$, TFCE-corrected) in AD compared to $\mathrm{HC}$ (yellow), in $\mathrm{MCl}$ compared to $\mathrm{HC}$ (orange), and in both $\mathrm{AD}$ and $\mathrm{MCl}$ compared to $\mathrm{HC}$ (red). Note that the cerebellum presents large areas of increased $\mathrm{FC}$ in $\mathrm{MCl}$, suggesting active compensatory mechanisms.
$17.76 \%$ of the total area of reduced $\mathrm{FC}$ in $\mathrm{AD}$ was also reduced in MCI, spreading over several networks. Although broad cortical regions were involved, the largest FC reductions were localized in the prefrontal cortex (i.e., PreF, including inferior, middle and superior frontal gyri) and in the precentral cortex (PreC), therefore involving primarily the FCN, both in $\mathrm{AD}(25.06 \%$ of the total extension of the FCN) and MCI (13.90\% of the total extension of the FCN), (Figure 2).

\section{Increased FC in multiple networks}

Several RSNs presented an FC increase both in AD (for a total of 1711 voxels affected) and MCI (for a total of 1627 voxels affected). The $37.22 \%$ (627 voxels) of the total area of increased FC in AD was also increased in MCI, spreading over several networks. An interesting observation is that the RSNs showing a FC increase in patients compared to HCs the most included part of the cerebellum (C), which also showed voxels of increased FC (Figure 3).

\section{GLOBAL NETWORK ANALYSIS \\ Ranking of $\mathrm{gFC}$ changes in $\mathrm{MCl}$ and $\mathrm{AD}$}

In order to better identify the patterns of alterations within the RSNs, we assessed the behavior of the $g F C$ index for each of the four contrasts. Results, which are summarized in Figure 4, lead to the identification of 6 RSN with prototypical patterns:
(1) The FCN showed the largest $g F C$ reduction, both in MCI and $\mathrm{AD}$, and no increase at all.

(2) The AIN, on the contrary, showed no $g F C$ reduction at all, but a large increase in both MCI and AD.

(3) The DMN showed the second largest $g F C$ reduction, twice as large in $\mathrm{AD}$ than in MCI, but at the same time it showed also the largest increase of $g F C$ in both $\mathrm{AD}$ and MCI.

(4) The BGN showed a reduction of $g F C$ only in $\mathrm{AD}$ but not in $\mathrm{MCI}$, while it also showed an increase of $g F C$ in both cases.

(5) The LVN showed mainly an increase of $g F C$ in AD.

(6) The CBLN showed just a minor reduction of $g F C$ in $\mathrm{AD}$ but a remarkable increase of $g F C$ in MCI only.

The other RSNs showed patterns similar to or between the prototypes identified here, with more modest alterations of $g F C$ than these six networks. In particular, it is worth noticing that the SN has a pattern similar to the CBLN, where a reduction of $g F C$ was seen in $\mathrm{AD}$ but not so much in MCI, while an increased $g F C$ was observed mainly in MCI. These alterations, though, are less than half in size than similar changes in $g F C$ in the CBLN.

\section{INDIVIDUAL NETWORK ANALYSIS \\ Patterns of rgFC changes in RSNs of $\mathrm{MCl}$ and AD}

Once the 6 RSNs were identified through the $g F C$ ranking, these were investigated in terms of the involvement of specific 


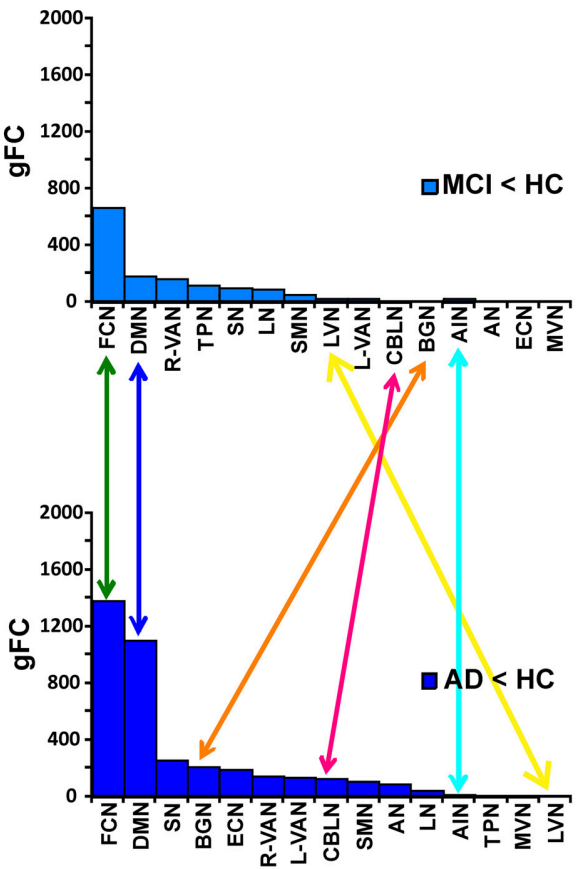

FIGURE 4 | Ranking of $\boldsymbol{g} \mathbf{F C}$ changes in $\mathbf{M C l}$ and $\mathbf{A D}$. In order to better identify the patterns of $g F C$ changes within the networks, for each contrast $(\mathrm{MCl}<\mathrm{HC}, \mathrm{MCl}>\mathrm{HC}$ on the top; $\mathrm{AD}<\mathrm{HC}, \mathrm{AD}>\mathrm{HC}$ on the bottom of the picture) we ranked the RSN alterations in terms of their decreasing $g F C$. We identified 6 different prototypical patterns: (1) FCN (green arrows) showed the largest $g F C$ reduction, both in $A D$ and $\mathrm{MCl}$ and no increase in any conditions; (2) AIN (turquoise arrows) showed no $g F C$ reduction, either in $A D$ or $\mathrm{MCl}$, but it showed a large increase in both

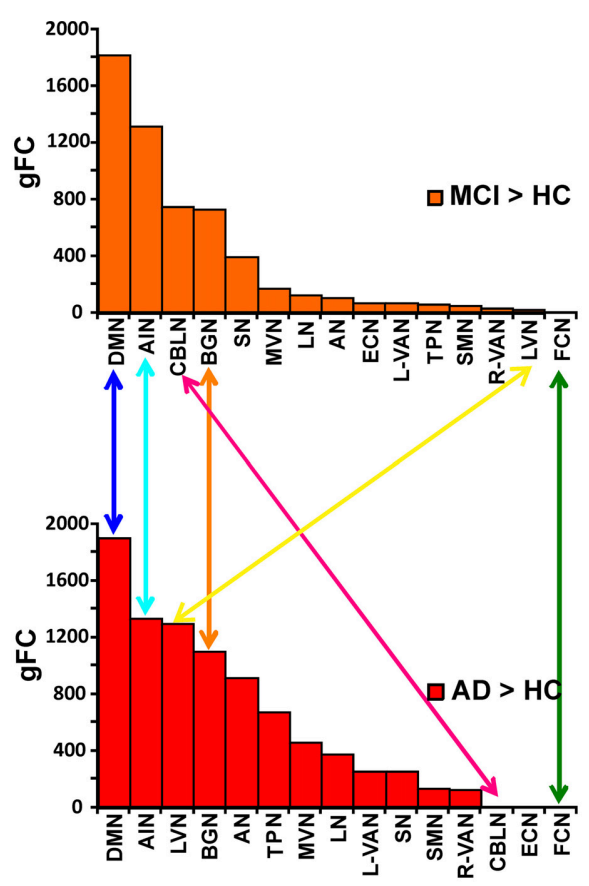

conditions; (3) DMN (blue arrows) showed the second largest $g F C$ reduction, both in $A D$ and $\mathrm{MCl}$, but at the same time it showed also the largest increase in both conditions; (4) BGN (orange arrows) shows a reduction in $A D$ but not in $\mathrm{MCl}$, while it also shows an increase in both cases; (5) CBLN (magenta arrows) shows just a minor decrease in AD but a remarkable increase in $\mathrm{MCl}$ only; (6) LVN (yellow arrow) shows $g F C$ increase in both $A D$ and $\mathrm{MCl}$, with stronger effect in $A D$. At the same time it showed a small $g F C$ reduction in $\mathrm{MCl}$, but not in $A D$. subcortical regions with the $\mathrm{r} g F C$ index defined above in the methods. Figure 5 gives a visual assessment of the involvement of each sub-area of the cortex in terms of decreased or increased rgFC.

In particular:

(1) In the FCN, in both $\mathrm{AD}$ and MCI, a decrease of the $\mathrm{rgFC}$ was mainly in the prefrontal cortex (PreF), with a small reduction also in the temporal cortex $(\mathrm{T})$. This effect was stronger in $\mathrm{AD}$ than $\mathrm{MCI}$, i.e., $\mathrm{r} g F C$ changes in $\mathrm{AD}$ are twice the size of $\mathrm{MCI}$ changes. In $\mathrm{AD}$, there was a further area of $\mathrm{r} g F C$ reduction corresponding to the limbic cortex (L). No rgFC increase was detected in any area of this network.

(2) In the AIN, very small areas of $\mathrm{rgFC}$ decrease were found in the temporal cortex $(\mathrm{T})$ in $\mathrm{AD}$ as well as in the cerebellar cortex (C) in MCI. On the other hand, the AIN was indeed the top-scoring network, after the DMN, when ranking them by their overall $g F C$ increase (Figure 4). The regional assessment showed that the $\mathrm{r} g F C$ increase occurred predominantly in the temporal cortex $(\mathrm{T})$ in both $\mathrm{AD}$ and $\mathrm{MCI}$ with stronger effects in AD than MCI, but also in the cerebellum (C), prefrontal cortex (PreF) and limbic cortex (L) in both $\mathrm{AD}$ and in MCI.

(3) In the DMN, alterations both in terms of decreased and increased $\mathrm{r} g F C$ values affected several cortical areas. We found that $\mathrm{rgFC}$ decreased mainly in the parietal $(\mathrm{P})$ and occipital cortices $(\mathrm{O})$, including cuneus and precuneus areas, with stronger alterations in $\mathrm{AD}$ than MCI. Smaller $\mathrm{rgFC}$ reductions were found in the limbic cortex $(\mathrm{L})$ and in the prefrontal cortex (PreF) in both $\mathrm{AD}$ and $\mathrm{MCI}$, where it also extended to precentral cortex (PreC). In $\mathrm{AD}$, there was a further area of $\mathrm{rgFC}$ reduction in the cerebellum (C) involving the left VI-V lobes and culmen. On the other hand, the DMN showed the areas of major $\mathrm{r} g F C$ increase in the limbic (L) and in the parieto-occipital cortices ( $\mathrm{P}$ and $\mathrm{O}$ ) in both $\mathrm{AD}$ and MCI, with stronger alterations in MCI than AD. Other areas of increased $\mathrm{rgFC}$ were found in the prefrontal cortex (PreF) in both $\mathrm{AD}$ and $\mathrm{MCI}$, with stronger effects in $\mathrm{AD}$ than MCI, and in the temporal cortex $(\mathrm{T})$ of the $\mathrm{AD}$ group.

(4) In the BGN, the r $g F C$ decreased only in the temporal cortex (T) in $\mathrm{AD}$. The $\mathrm{r} g F C$ increased mainly in the temporal cortex (T) and in the prefrontal cortex (PreF) in both AD and MCI. These effects were stronger in $\mathrm{AD}$ than MCI. Further areas of increased $\mathrm{r} g F C$ were observed in $\mathrm{AD}$ in the parietal $(\mathrm{P})$ and occipital $(\mathrm{O})$ cortices.

(5) In the LVN, a small area of $\mathrm{r} g F C$ decrease was observed in the occipital cortex $(\mathrm{O})$ in $\mathrm{MCI}$. No $\mathrm{r} g F C$ reductions were observed instead in $\mathrm{AD}$. On the other hand, the $\mathrm{r} g F C$ increase was shown mainly in the occipital cortex $(\mathrm{O})$ of $\mathrm{AD}$. In 


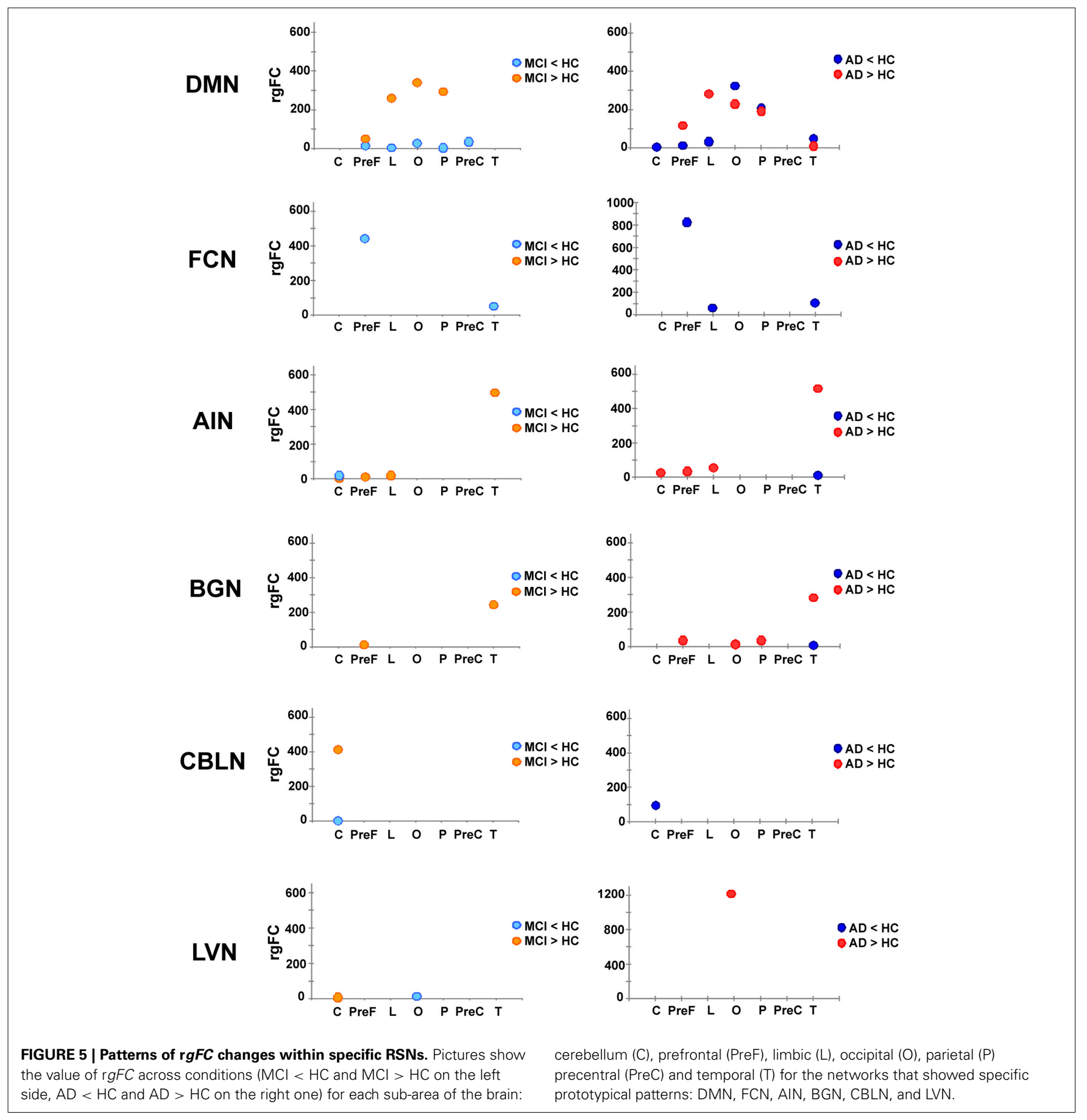

$\mathrm{MCI}$, there was only a small area of $\mathrm{rgFC}$ increase in the cerebellum (C).

(6) In the CBLN, all changes happened at cerebellar level (C) because this network does not involve any other region of the cortex. Here, the r $g F C$ decrease was greater in $\mathrm{AD}$ than $\mathrm{MCI}$, while the $r g F C$ increase was strong (third RSN in the ranking by $g F C$ increase) and present only in MCI.

Although the meaning of $\mathrm{rgFC}$ increase or decrease in physiological terms is not fully clarified (see Discussion), there was a clear relationship between $\operatorname{rgFC}$ changes and the worse phase of the pathology.

\section{Nodes of alterations}

If we consider all the RSNs obtained with the dual regression analysis it is evident that there are several areas that functionally contribute to more than one network and as such can be considered as nodes or hubs. In particular, the sub-area analysis of $\mathrm{r} g F C$ alterations has highlighted three possible nodes, which are involved in alterations of several networks. These are: the 


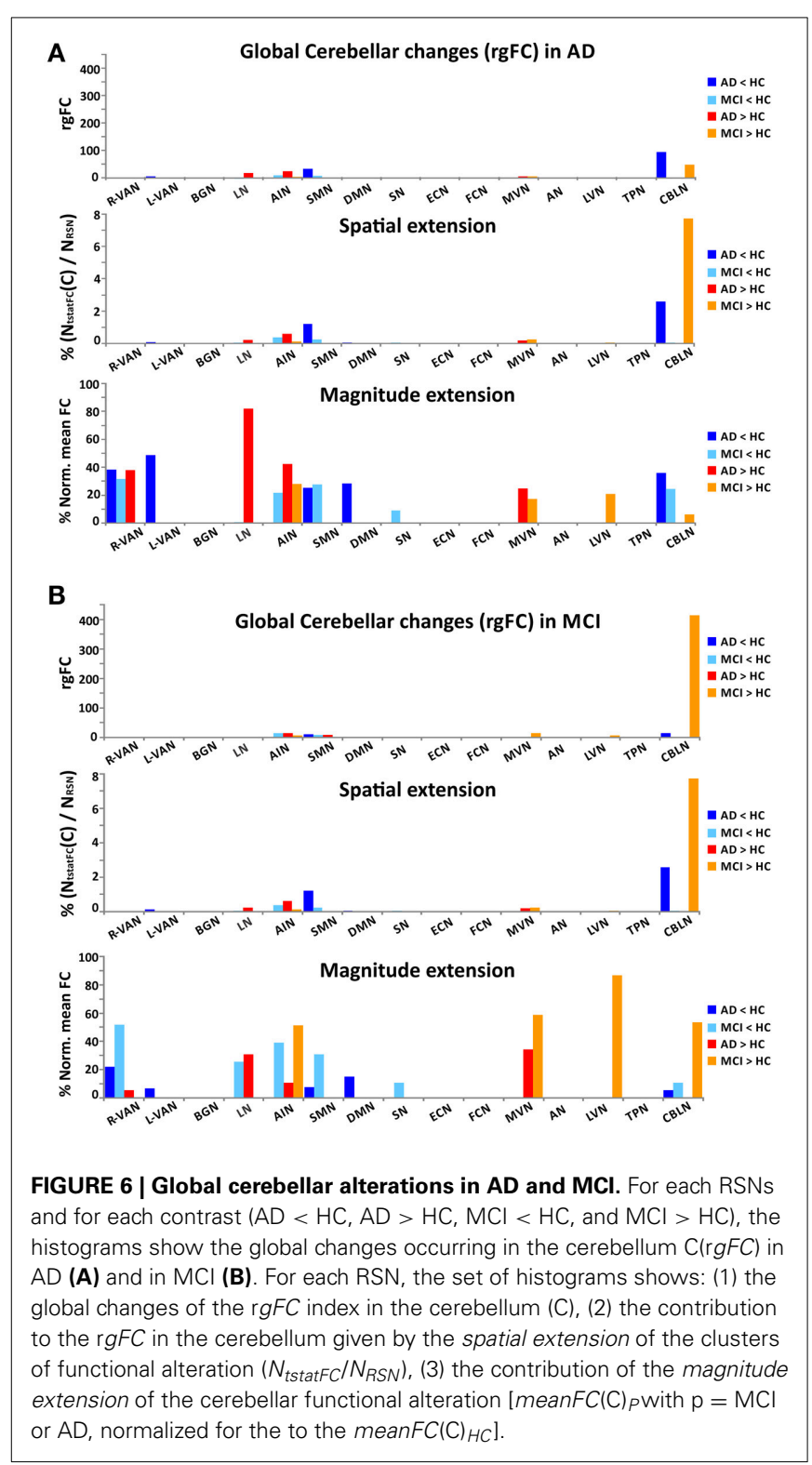

prefrontal cortex (PreF: 2.22\% of prefrontal alteration in $\mathrm{AD}$ and $1.09 \%$ in $\mathrm{MCI}$ ), the mesial-temporal cortex (T: $2.33 \%$ of mesial temporal alteration in $\mathrm{AD}$ and $1.05 \%$ in $\mathrm{MCI}$ ) and the parietal cortex (P: $1.76 \%$ of parietal cortex alterations in $\mathrm{AD}$ and $1.11 \%$ in $\mathrm{MCI})$.

Although the cerebellum (C) doesn't emerge as one of the principal nodes of $\mathrm{rgFC}$ alteration (C: $0.41 \%$ of cerebellar alteration in $\mathrm{AD}$ and $0.79 \%$ in $\mathrm{MCI}$ ), it is often involved both in $\mathrm{MCI}$ and $\mathrm{AD}$ showing altered $\mathrm{rgFC}$ values across several RSNs (Figure 6). There were, in fact, 10 RSNs presenting areas of $\mathrm{rgFC}$ changes in the cerebellum (C): the CBLN, the DMN, the AIN, the $\mathrm{SN}$, the MVN, the LN, the VANs, the SMN, and the LVN. MCI showed the major areas of $\mathrm{rgFC}$ increase in DMN, AIN and CBLN with the most prominent $\mathrm{rgFC}$ increase in the $\mathrm{DMN}$. AD showed areas of $\mathrm{rgFC}$ decrease particularly in DMN, SN, CBLN, VANs, and SMN but also a large area of $\mathrm{r} g F C$ increase in the DMN.

\section{VBM STRUCTURAL CHANGES}

VBM analysis in AD subjects (compared to $\mathrm{HC}$ ) showed large areas of GM atrophy mainly in the left and right hippocampi, left amygdala, parahippocampal gyrus, uncus, inferior, middle, superior, and transverse temporal gyri (Figure 7A), as well as in smaller areas in the right amygdala and precuneus. VBM analysis in MCI subjects (compared to HC) showed areas of GM atrophy mainly in left and right hippocampi and in the left amygdala, the parahippocampal gyrus, the precuneus, and the superior temporal gyrus (Figure 7B). As a whole, GM atrophy was more extended in $\mathrm{AD}\left(68,736 \mathrm{~mm}^{3}\right)$ than $\mathrm{MCI}\left(8,184 \mathrm{~mm}^{3}\right)$ with a ratio approximately of $8: 1$.

It should be noted that GM atrophy was significantly detected in a large area of the bilateral mesial-temporal cortex $(p<$ 0.001 , FDR-corrected) and that, although both $\mathrm{AD}$ and $\mathrm{MCI}$ were atrophic in the superior temporal gyrus and in the mesial-temporal areas (left and right hippocampi, amygdala, and parahippocampal gyrus), AD presented larger clusters of atrophy than MCI. In particular AD subjects compared to MCI were more atrophic in the superior and middle temporal gyri. These results substantially confirmed previous reports (Ries et al., 2008; Ferreira et al., 2011) and revealed that areas of GM atrophy overlap at the $6.8 \%$ with the DMN, although only a small portion $(1.65 \%)$ of this areas of GM atrophy were found co-localized with FC alterations.

In particular, the comparison of structural and functional changes in $\mathrm{AD}$ subjects (compared to $\mathrm{HC}$ ) showed that GM atrophy was consistent with the FC decrease observed in the BA 20, 21 , and 22 in the inferior, middle and superior temporal gyri (T), in the right parahippocampal gyrus, in the BA 10 in the prefrontal areas (PreF), in the cuneus and in the left precuneus (Figure 7E). Similarly, the comparison of structural and functional changes in $\mathrm{MCI}$ subjects (compared to $\mathrm{HC}$ ) showed that GM atrophy is consistent with the FC decrease observed in the middle and inferior temporal gyrus $(\mathrm{T})$, although it should be noted that the overlapping between atrophy and FC changes resulted very limited (see Figure 7F).

\section{CORRELATION BETWEEN FUNCTIONAL CONNECTIVITY AND THE CLINICAL AND NEUROPSYCHOLOGICAL STATE}

In order determine whether a relationship existed between the complex changes in brain $\mathrm{rgFC}$ and the pathological state of the $\mathrm{AD}$ and $\mathrm{MCI}$ subjects, a cross-correlation matrix was generated (Figure 8).

\section{Correlations in AD (compared to HC)}

In $\mathrm{AD}$ (compared to HC) the MMSE, SF, CF, MP, TMT-A, TMT$\mathrm{B}$, and ROCF-rec indexes were found to correlate $(p<0.001)$ with the mean $\mathrm{r} g F C$ value in areas of reduced $\mathrm{rgFC}$ in the PreF, PreC, $\mathrm{T}$ and $\mathrm{L}$ areas of multiple networks (Figure 8A). The MMSE showed the major number of significant correlations with reduced $\mathrm{r} g \mathrm{FC}$ over some of the most relevant brain areas for $\mathrm{AD}$ (PreF, PreC, T, and L). In detail, the MMSE inversely correlated $(p<0.001)$ with reduced $\operatorname{rgFC}$ in the DMN $(\operatorname{PreF})$ and $\mathrm{DMN}(\mathrm{L})$, in the $\mathrm{ECN}(\mathrm{PreF})$ and $\mathrm{ECN}(\mathrm{PreC})$ and in the R-VAN(T), the $\mathrm{AN}(\mathrm{T})$ and the $\mathrm{LN}(\mathrm{T})$. Areas of decreased $\mathrm{rgFC}$ in the $\mathrm{DMN}(\mathrm{L})$ showed a strong inverse correlation with both 1/TMT-B and 


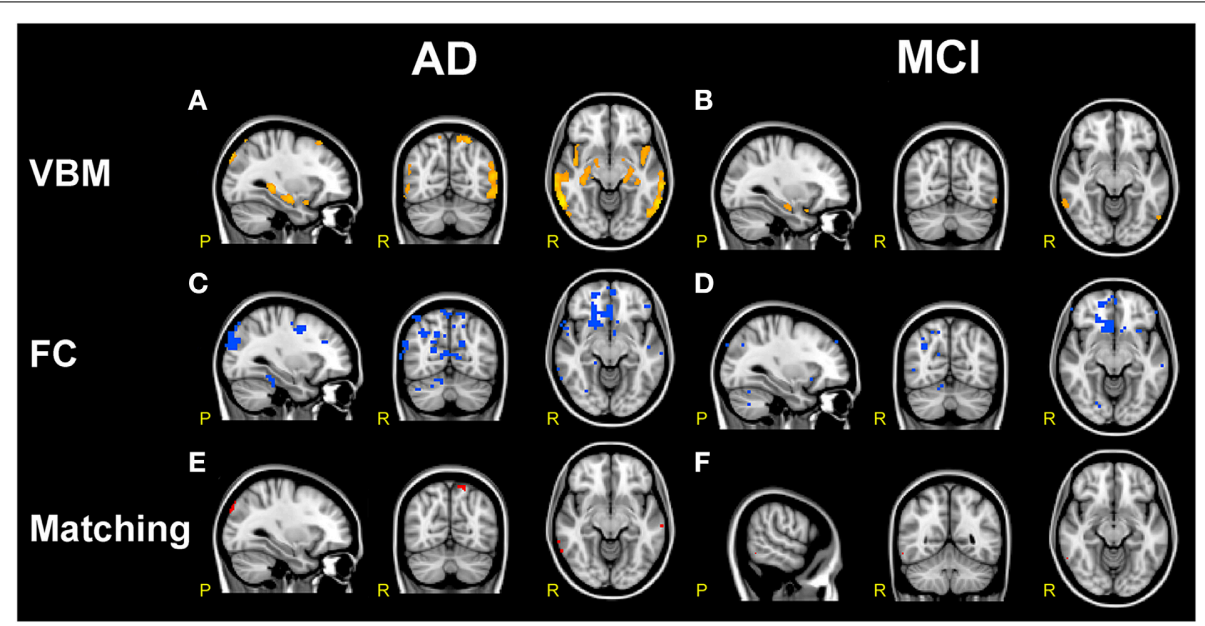

FIGURE 7 | Comparison of structural and functional changes in AD and MCI. (A,B) VBM analysis. AD subjects compared to $H C(\mathbf{A})$ show large areas of $\mathrm{GM}$ atrophy (orange) in bilateral mesial-temporal network. $\mathrm{MCl}$ subjects compared to $\mathrm{HC}$ (B) show sparse areas of GM atrophy (orange) in left and right hippocampus, left amygdala, parahippocampal gyrus, precuneus and superior temporal gyrus. AD show more extended areas of atrophy than $\mathrm{MCl}$.
(C,D) FC analysis. Both $\mathrm{AD}$ and $\mathrm{MCl}$ compared to $\mathrm{HC}$ show large clusters of $\mathrm{FC}$ reductions (blue) mainly localized in the prefrontal areas involving $V A N$, DMN, DAN, FCN, SN, and SMN, with more extended areas of $F C$ reductions in $\mathrm{AD}$ than $\mathrm{MCl}$. (E,F) VBM/FC matching. Matching $V B M$ and $\mathrm{FC}$ maps of $\mathrm{FC}$ reduction for $\mathrm{AD}$ compared to $\mathrm{HC}$ and $\mathrm{MCl}$ compared to $\mathrm{HC}$. The overlapping between atrophy and FC changes (red clusters) is very limited.
1/TMT-A [which, also inversely correlated with reduced $\mathrm{r} g F C$ in the L-VAN(PreF)]. Both the SF and the CF inversely correlated with decreased $\mathrm{r} g F C$ in the $\mathrm{LN}(\mathrm{T})$, while the MP showed a strong inverse correlation with reduced $\mathrm{r} g F C$ of the $\mathrm{AN}(\mathrm{T})$. Finally, the ROCF-rec was found to inversely correlate with decreased $\mathrm{r} g \mathrm{FC}$ in the $\mathrm{LN}(\mathrm{T})$ and positively correlate with the $\mathrm{ECN}(\mathrm{PreC})$.

Moreover in $\mathrm{AD}$, the MMSE, MP, ROCF-rec and the CF showed a major number of correlations with increased $\mathrm{r} g \mathrm{FC}$ over different RSN brain areas (Figure 8C). The MMSE inversely correlated with increased $\mathrm{rgFC}$ in the $\operatorname{AIN}(\mathrm{C}), \operatorname{AIN}(\mathrm{L})$ and $\operatorname{AIN}(\mathrm{T})$, in the $\mathrm{LN}(\mathrm{T})$ and $\operatorname{SMN}(\mathrm{T})$, and in the $\operatorname{DMN}(\mathrm{O})$. An inverse correlation was also observed between the $\mathrm{CF}, \mathrm{MP}$, and ROCF-rec and increased $\mathrm{r} g F C$ in the $\mathrm{AIN}(\mathrm{T})$. The ROCF-rec inversely correlated also with increased $\mathrm{r} g F C$ in the $\mathrm{LN}(\mathrm{T})$ and the $\mathrm{SMN}(\mathrm{T})$. Moreover, increased $\mathrm{r} g F C$ in the $\operatorname{DMN}(\mathrm{O})$ and the $\operatorname{MVN}(\mathrm{O})$ inversely correlated with the MP. Moreover the MP and the 1/TMT-B inversely correlated with increased $\mathrm{rgFC}$ in the $\mathrm{LN}(\mathrm{C})$. A positive correlation was observed instead between all neuropsychological tests and the $\mathrm{MVN}(\mathrm{C})$, with a particularly strong positive correlation between the MMSE and the CF indexes with an increased $\mathrm{r} g \mathrm{FC}$ in the $\mathrm{MVN}(\mathrm{C})$.

\section{Correlations in MCI (compared to HC)}

In $\mathrm{MCI}$, a reduced number of significant correlations, considering a $p<0.001$, were observed between the neurological indexes (MMSE, CF, MP, ROCF-rec, and SF) and reduced rgFC areas over the brain compared to $\mathrm{AD}$ as shown by a reduced matrix size (Figure 8B compared to Figure 8A). In detail, in MCI areas of reduced $\mathrm{r} g F C$ in the $\mathrm{L}-\mathrm{VAN}(\mathrm{T})$ showed an inverse correlation with the MMSE and the CF. Significant inverse correlations were observed between the MP and reduced $\mathrm{rgFC}$ in the $\mathrm{DMN}(\mathrm{O})$, as well as between the ROCF-rec and reduced $\mathrm{rgFC}$ in the $\mathrm{SMN}(\mathrm{P})$. A positive correlation was found instead between the SF and reduced $\mathrm{rgFC}$ in the $\mathrm{CBLN}(\mathrm{C})$.
Furthermore, in MCI the 1/TMT-A, 1/TMT-B, MMSE, CF, $\mathrm{MP}$, and the ROCF-rec showed at least a significant correlation with increased $\mathrm{rgFC}$ over different RSN areas (Figure 8D). In particular, strong inverse correlations were observed between the ROCF-rec and increased $\mathrm{r} g F C$ in the $\mathrm{MVN}(\mathrm{T})$, as well as between the MMSE and the SMN(T). The 1/TMT-A also anti-correlated with increased $\mathrm{rgFC}$ in the $\mathrm{SN}(\mathrm{T})$, while the MP showed strong inverse correlation with increased $\mathrm{rgFC}$ in the $\mathrm{SMN}(\mathrm{T})$ and in the $\mathrm{LN}(\mathrm{P})$. The CF showed instead a positive correlation with increased $\mathrm{rgFC}$ in the $\mathrm{MVN}(\mathrm{C})$ and in the $\mathrm{LN}(\mathrm{PreF})$, as well as the 1/TMT-B was found to correlate positively with the AIN(C).

Comparing trends of correlations between mean $\mathrm{r} g F C$ in areas of increased $\mathrm{rgFC}$ and neuropsychological scores in $\mathrm{AD}$ and $\mathrm{MCI}$, three are the areas that are emerging as being correlated in both groups of patients (Figures 8C,D): $\operatorname{MVN}(\mathrm{C}), \operatorname{SMN}(\mathrm{T})$, and $\mathrm{AIN}(\mathrm{C})$. The $\mathrm{MVN}(\mathrm{C})$ seems to present a similar trend in MCI and $\mathrm{AD}$ for all tests. The $\mathrm{SMN}(\mathrm{T})$ instead shows an inverse correlation between $\mathrm{r} g F C$ increase and MMSE in both groups, while $\mathrm{AIN}(\mathrm{C})$ shows a positive correlation between $\mathrm{rgFC}$ increase and $\mathrm{CF}$ scores in MCI and an inverse correlation between $\mathrm{r} g \mathrm{FC}$ and $\mathrm{CF}$ scores in AD. Similarly there is a positive correlation between the $r g F C$ increase and MMSE in MCI, which instead is an inverse correlation in $\mathrm{AD}$.

\section{CORRECTION FOR RELATIVE GM VOLUME}

All the clusters of FC reductions and FC increases, described so far, survived statistical thresholding ( $p<0.05$, TFCE-corrected) when correcting for GM atrophy.

\section{DISCUSSION}

A hallmark of $\mathrm{AD}$ and MCI in rs-fMRI studies is the FC reduction in the DMN, which was confirmed by our findings that showed a reduced FC connectivity in a large temporo-parietal ( $\mathrm{T}$ and $\mathrm{P}$ ) area, centered in the cuneus, precuneus and the posterior 


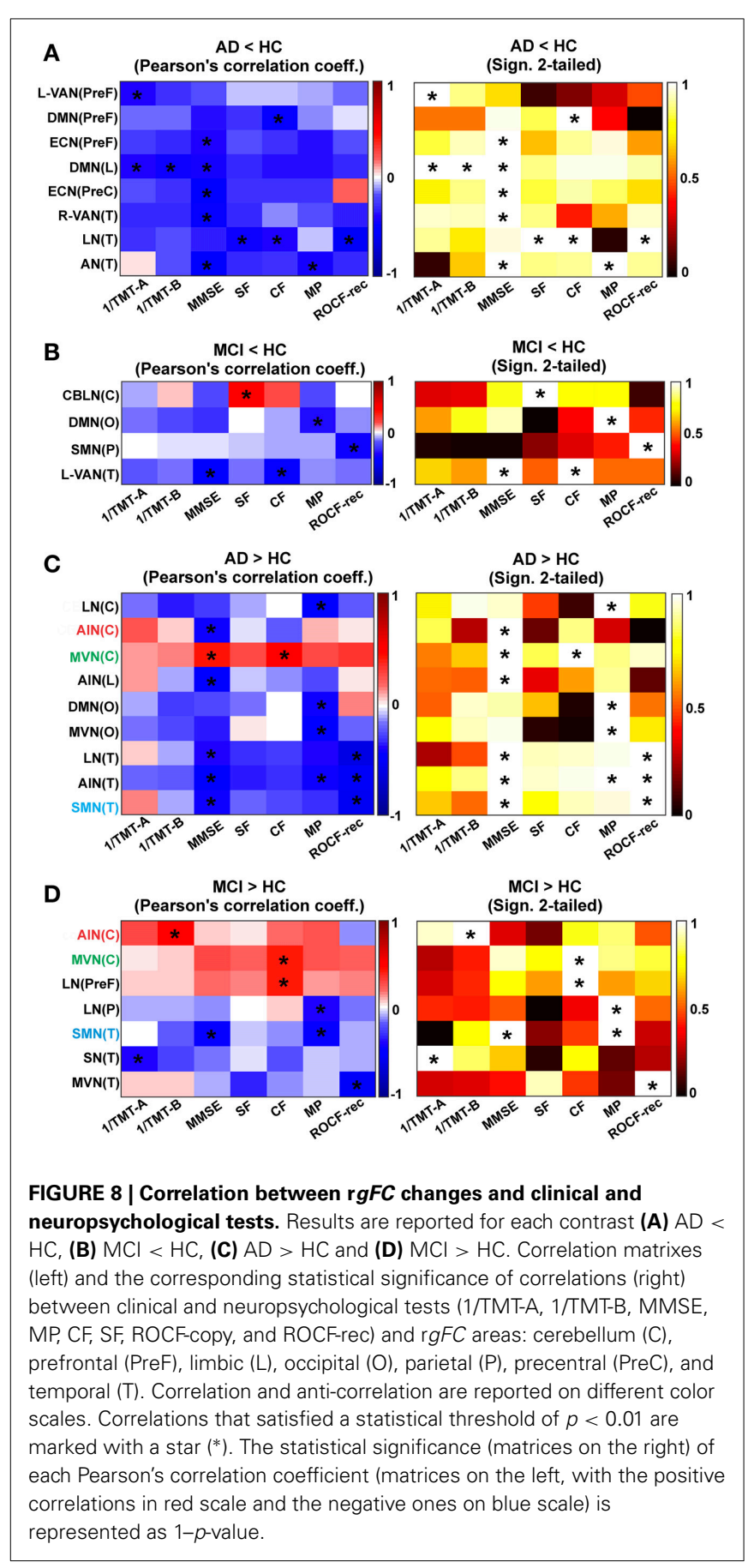

cingulate cortex, in $\mathrm{AD}$ and in MCI, although in MCI areas of FC disruption were found less extended $(20.05 \%$ of the DMN was affected by FC reduction in $\mathrm{AD}$, while only $3.17 \%$ in $\mathrm{MCI}$ ).

In this paper we presented a global approach for a comprehensive assessment of alterations in FC of all RSNs and applied it to a pilot study of dementia patients. The introduction of a global parameter (the $g F C$ index) was driven by the need to summarize both FC changes and voxels extension of such changes for each RSN and allowed us to (i) create a ranking of all RSNs, (ii) highlight which are the main RSNs undergoing significant patterns of alterations and (iii) evaluate the contribution of areas of the cerebral cortex and cerebellum to such changes in different pathological states.

The main observation in this pilot study is that multiple RSNs, rather than just DMN, are modified in $\mathrm{AD}$ and $\mathrm{MCI}$ patients presenting both patterns of decreased and increased FC changes which could be identified in six different prototypical RSNs. Furthermore, although a widespread FC change involved to some extent all the $15 \mathrm{RSN}$ identified (namely $A N, E C N$, VANs, LN, DMN, SMN, BGN, AIN, SN, FCN, LVN, MVN, TPN, and $C B L N$ ) the sub-area analysis that we performed revealed the emergence of alterations in specific nodes (or hubs) including primarily the prefrontal cortex (PreF), the mesial-temporal cortex $(\mathrm{T})$, and the parietal cortex $(\mathrm{P})$. We also acknowledge the extensive involvement of the cerebellum (C) in several RSN disruptions. In addition to support a functional disconnection between prefrontal cortex (PreF) and hippocampus (T) (Wang et al., 2006; Zhang et al., 2009), our data revealed that both $\mathrm{AD}$ and MCI showed areas in which $g F C$ was either reduced or increased, with a prevalent $g F C$ decrease in $\mathrm{AD}$ but also an equally relevant $g F C$ increase in both $\mathrm{AD}$ and $\mathrm{MCI}$ (see Figure 4). Noticeably the $g F C$ increase affected also the DMN. Finally, these $g F C$ changes correlated with the neuropsychological status of patients.

When dealing with FC increases, however measured (through the standard FC parameter or $g F C$ or $\operatorname{rgFC}$ ), it is tempting to interpret data as compensatory mechanisms taking place in $\mathrm{MCI}$ while network disruption prevailing in AD. This first justification is challenged in details below as it may be too simplistic in the presence of a complex pathophysiology such as in dementia. Interestingly, both the FC reductions and the FC increases survived statistical thresholding ( $p<0.05$, TFCE-corrected) when correcting for GM atrophy, indicating that the FC alterations we observed are likely to be independent from global GM atrophy. Also, FC changes overlapped only minimally with local GM reductions as shown by the VBM analysis.

While these results need confirming in larger clinical studies, they raise important issues such as: What is the nature of FC alterations in the absence of morphological alterations? Why do some areas show increased FC while others show decreased FC? What is the meaning of the cerebellum FC changes? Are there any specific correlations between the areas showing FC changes and the neuropsychological state of patients?

\section{FUNCTIONAL ALTERATION IN AREAS WITHOUT SIGNIFICANT CORTICAL ATROPHY}

As expected, GM atrophy was more extended in AD than MCI with a ratio approximately of 8:1. Both GM atrophy and FC reductions in $\mathrm{AD}$ and $\mathrm{MCI}$ affected in particular the prefrontal cortex (inferior, middle and superior gyri) and a large mesialtemporal-parieto-occipital (T-P-O) area (including hippocampus, parahippocampal gyrus, precuneus, cuneus, and amygdala). These observations are in line with others showing similar profiles in neuropathology, atrophy, metabolism and FC in the cortical surface of AD patients (Buckner et al., 2005; Ries et al., 2008; Ferreira et al., 2011). However, VBM analysis revealed that areas of reduced FC only partially overlapped with GM atrophy both in $\mathrm{AD}$ (333 voxels equals to the $3.78 \%$ of the GM atrophy area) and MCI ( 1 voxel equals to the $0.09 \%$ of the GM atrophy area). 
Since there is a clear relationship between neuropathological signs (amyloid plaques and tau tangles) in the prefrontal cortex (PreF) and mesial-temporal ( $\mathrm{T}$ ) lobe and reduced cognitive performance in $\mathrm{AD}$, the emergence of $\mathrm{FC}$ alterations in regions classically devoid of neuropathological alterations (e.g., several other cortical areas and the cerebellum) is puzzling. A possible explanation is that primary damage causes a subsequent derangement of functional relationships between multiple areas, a fact that should not be surprising as we are dealing with large-scale networks in which different areas are functionally interconnected. The prefrontal cortex and mesial-temporal lobe are hubs taking part in multiple functional networks, and their alteration might reverberate into widespread changes. Whatever the sign and mechanism of FC changes, a generalized alteration in RSNs is akin with the proposal that central neurodegenerative diseases, including $\mathrm{AD}$ eventually drive into a dysfunctional state large-scale networks extending beyond the initial core of neurodegeneration (Seeley et al., 2009).

\section{SEEKING FOR PATTERNS OF FC CHANGE IN RSNs OF MCI AND AD}

Once moving beyond the concept that $\mathrm{AD}$ and MCI are characterized mainly by DMN changes, and once realizing that almost all the RSNs show alterations consisting of either an FC increase, decrease or both, the search for meaningful alteration patterns becomes complicated by the intersection of RSNs in multiple nodes. Moreover, the presence of FC changes that can differ in amplitude as well as spatial extent can complicate interpretation. We have assumed that both the absolute change of FC value and the extension of the change have a major contribution into network disruption. Within this framework we have introduced the generalized FC index $(g F C)$, which has revealed general patterns of FC alterations summarized as follows: (i) the $g F C$ decrease was about twice as large in $\mathrm{AD}$ then $\mathrm{MCI}$, (ii) the $g F C$ increase was about four times larger than the $g F C$ decrease, (iv) the $g F C$ increase was extensive both in MCI and AD.

Understanding how these FC changes could be generated is challenging. An hypothesis could be that changes start from the DMN and FCN, in which the FC decrease may reflect primary structural alterations already present in MCI and persisting in AD. Passing through the nodes of the DMN and FCN, the functional alterations may reverberate into other networks. Since there is a critical worsening of symptoms in AD compared to MCI, an FC decrease may correlate with reduced functionality. Given the extent of $g F C$ increase in $\mathrm{AD}$, as well as $\mathrm{MCI}$, the interpretation of such an increase requires thoughts. On one hand, functional compensation may exploit the pre-existing neural reserve through plasticity and it would be easy to justify as a mechanism particularly active in MCI. Then, either due to hypo-activation or to a more extended micro-structural damage, more and more networks could run into a hypo-functional state reflecting the condition of $\mathrm{AD}$ with a larger decrease of $g F C$. This may be true for the CBLN, showing a net switch from increased $g F C$ to decreased $g F C$ when considering MCI or $\mathrm{AD}$, and for the $\mathrm{BGN}$, in which $g F C$ reduction appears just in $\mathrm{AD}$ patients. On the other hand though, some RSN like the AIN show an increase $g F C$ in both MCI and $\mathrm{AD}$. This could be interpreted as a compensatory mechanism typical of MCI, but still surviving even in patients with advanced pathology such as AD. Although this hypothesis is attractive for its simplicity, the fact that $g F C$ increase was even greater in $\mathrm{AD}$ than MCI suggests the possibility of a more complex scenario, in which functional degeneration and compensation may evolve dynamically presenting different patterns in different stages of the disease (Zhang et al., 2009; Bai et al., 2011a). An alternative interpretation of increased $g F C$ in both MCI and $\mathrm{AD}$ is that it represents, at least to some extent, hyper-synchrony and phase locking, thereby reducing mutual information transfer through network nodes (Borst and Theunissen, 1999). Consistent with this hypothesis is the finding of diffused increase of spectral power in the EEG delta band of $\mathrm{AD}$ patients, which is also index of disease progression (Babiloni et al., 2004, 2014). In this case, the hypothesis of a compensatory mechanism may be erroneous. Rather, increased $g F C$ may be an unsuccessful attempt of compensation, therefore may be interpreted itself as a disease expression.

To further evaluate these hypotheses, multidisciplinary approaches might be used; in particular positron emission tomography (PET) imaging for measures of glucose metabolism and combined electrophysiological and fMRI experiments should be devised. It would also be of interest to evaluate whether these FC changes are accompanied by corresponding structural changes in the tracts connecting cortical areas and the cerebellum.

\section{INVOLVEMENT OF THE CEREBELLUM IN MCI AND AD}

The functional involvement of the cerebellum in $\mathrm{AD}$ is in line with the observations that MCI patients exhibit altered cerebellothalamo-cortical activations during Stroop tests (Kaufmann et al., 2008), abnormal thalamo-cortico-cerebellar connections (Teipel et al., 2008) and significantly lower FC in a network involving hippocampus, prefrontal lobe, temporal lobe and parietal lobe (Bai et al., 2009). The cerebellum was also reported to undergo degenerative changes in $\mathrm{AD}$, showing atrophy of the posterior cerebellar region associated with impaired cognitive performance. Therefore, our result supports the hypothesis that the cerebellum is involved in different forms of cognitive impairment including MCI and AD (Thomann et al., 2008).

Since the cerebellum is typically devoid of amyloid plaques (Serrano-Pozo et al., 2011; Ni et al., 2013), the RSN changes involving the cerebellum may be explained, at least in part, by abnormal recruitment of neurons caused by a primary cerebrocortical change. Since in the cerebellum, and in particular the CBLN, a $g F C$ increase prevails in MCI while a $g F C$ reduction prevails in $\mathrm{AD}$, the former may reflect a reaction to the altered relationship with the cerebral cortex, while the latter may be a direct consequence of functional disruption. It is tempting to speculate that increased cerebellar $g F C$ takes part in a homeostatic mechanism that aims at limiting the progression of cognitive decline from MCI to AD, which can only be properly tested in longitudinal studies.

\section{CORRELATION OF FUNCTIONAL CONNECTIVITY CHANGES WITH THE NEUROPSYCHOLOGICAL STATE}

In line with MCI and AD clinical definitions, the major alterations observed at the neuropsychological level concerned attention and memory. These two functions are elaborated in extended brain networks involving the prefrontal cortex (PreF), mesial-temporal 
lobe (T) and cerebellum (C), which all showed altered activity in $D M N, F C N, V A N, L N, S M N, A I N, M V N, L V N, C B L N$. Among the tests, MMSE and MP and CF were those that showed the most significant correlations with $\mathrm{r} g F C$ changes. The MMSE showed the major number of significant correlations with most brain regions (PreF, PreC, T, $\mathrm{L}, \mathrm{O}$ and $\mathrm{C}$ in $\mathrm{AD} ; \mathrm{T}$ and $\mathrm{C}$ in $\mathrm{MCI}$ ). Moreover, the MMSE is also the only index to show more than one significant correlation $(p<0.001)$ with both $\mathrm{r} g F C$ increase and $\mathrm{r} g \mathrm{FC}$ decrease in the cerebellum, which is known to be strongly interconnected with both motor and non-motor (associative) areas, including PreF and $\mathrm{T}$ cortices (Houk and Wise, 1995; Schmahmann et al., 1999; Habas et al., 2009; Krienen and Buckner, 2009; Stoodley and Schmahmann, 2010; Stoodley, 2012; Stoodley et al., 2012; Keren-Happuch et al., 2014). The cerebellum is important for operations of timing, prediction and learning and for integrating them into processes of novelty/error detection, working memory and mental manipulation (D'Angelo and Casali, 2012). This allows the cerebellum to take part not just to motor control but also to attention switching, language processing, imagery, decision making and reasoning, consistent with a potential involvement in the pathophysiology of MCI and AD.

The fact that both $\mathrm{r} g F C$ increase and $\mathrm{r} g F C$ reduction correlate with MMSE straighten the hypothesis that both $\mathrm{r} g F C$ modification are expression of disease, possibly in different diseases phases. In each cerebral area, the initial dysfunction may correlate with an attempt of compensation and a $\mathrm{r} g F C$ increase. Later in the disease progression, in the same area, also affected by atrophy, the $\operatorname{rgFC}$ decrease may predominate. It is indeed interesting that the correlations between MMSE and $\mathrm{r} g F C$ increase are mainly inverse in $\mathrm{AD}$ while some areas, including cerebellar regions $[\mathrm{AIN}(\mathrm{C})$ and $\operatorname{MVN}(\mathrm{C})$ ] are showing a positive trend in MCI.

Comparing the matrices in Figure $\mathbf{8 C}$ and $\mathrm{d}$ at a glance it is easy to see that the matrix referring to correlations in $\mathrm{AD}$ is much more "blue" (inverse correlation) than the matrix for the MCI where almost half of the squares are "red" (positive correlation) and involve cerebellar areas. This could be an indication supporting the interpretation that an increased $\mathrm{r} g F C$ could be due to compensatory mechanisms in MCI but to phase locking of signal transmission within a network in AD. Interestingly, for example, there is a positive correlation between the $\mathrm{r} g F C$ increase in the $\mathrm{AIN}(\mathrm{C})$ and the CF score in MCI, while an inverse correlation in $\mathrm{AD}$.

\section{POSSIBLE LIMITATIONS OF THE PRESENT STUDY}

The results reported in the present investigation have to be confronted with some technical and methodological limitations. First of all, the relatively small sample size might have limited the statistical power to display changes in regional FC in $\mathrm{AD}$ and $\mathrm{MCI}$ groups. Moreover, the MCI population was a mixture of amnestic and non-amnestic subjects. Although we performed a strict quality control on our images, we recognize that our MCI population represents a late stage of this phase of the disease. Future studies should assess the RSNs changes in cohorts of prodromal dementia patients (Minati et al., 2014), performing also longitudinal studies to follow changes in RSNs of patients who convert or do not convert to AD. Further studies will also be needed to recruit a larger sample of patients, to subdivide amnestic from non-amnestic MCI subjects and to follow-up their clinical and MRI evolution in a longitudinal study. The MRI protocol was performed on a $1.5 \mathrm{~T}$ clinical scanner with clinical protocols, rather than sequence parameters optimized for a research study. Current state of the art scanners (e.g., 3T) would allow better acquisition protocols, for example acquiring smaller isotropic voxels, in similar scan times. Moreover, it remains to be seen whether the FC findings in $\mathrm{MCI}$ are related to their possible progression to $\mathrm{AD}$, providing a possible biomarker for cognitive dysfunction in $\mathrm{AD}$ related illness evolution. Further investigations are also needed, including possible electrophysiological measurements, to help assessing the theory of compensatory mechanisms or reduced mutual information to explain increased FC in both MCI and AD.

Recent studies of metabolic energy demand of the resting human brain suggest that glutamatergic function in the resting awake human brain is supported by uniformly high oxidative energy (Hyder and Rothman, 2012; Hyder et al., 2013). Moreover, Tomasi and colleagues (Tomasi et al., 2013) showed that BOLD signal fluctuations with larger amplitudes are localized in brain regions characterized by higher metabolism. They also suggest that the higher energy demands of brain communication that hinges upon higher connectivity could render brain hubs more vulnerable to deficits in energy delivery or utilization and help explain their sensitivity to neurodegenerative conditions, such as AD (Tomasi et al., 2013). Other studies suggested, indeed, that there are organized patterns of brain metabolism, and the associated metabolic activity might reflect the relevant brain functions between the brain regions involved (Wu et al., 2009; Di et al., 2012). The above reported evidence suggests that rs-fMRI on $\mathrm{AD}$ and $\mathrm{MCI}$ should be associated to FDG-PET acquisitions in order to compare and discuss FC alterations with regard to metabolic deficiencies. Therefore, in future it could be useful to adopt multi-modal approaches combining measures of FC with indexes of fiber integrity and quantitative measures of glucose metabolism.

\section{CONCLUSIONS}

We have successfully developed a strategy to assess multiple RSNs in terms of their FC changes (both in magnitude and spatial extension) as well as to evaluate contributions of different cortical regions to FC alterations. The picture that emerges from these rsfMRI results suggests that MCI and AD FC changes occur in hubs laying at the intersection of multiple RSNs (all the 15 RSNs identified in the study), rather than just in the DMN, and encompass both decreased and increased FC. These hubs are mostly located in the prefrontal and parieto-temporal cortices. The cerebellum is also involved in several changes, which might suggest that it participates as well as various cortical areas to compensatory processes, although the hypothesis of reduced mutual information cannot be discarded, especially in the advanced stage of the disease such as in $\mathrm{AD}$. Longitudinal studies on large patients groups are needed to investigate physiological meanings of changes and the correlation between RSN changes and disease development. The pilot study presented here, though, thoroughly demonstrated that the assessment of alterations in FC of multiple extended large-scale networks of the cerebral cortex and cerebellum using rs-fMRI deserves further attention and may provide new cues to 
examine the pathophysiology of neurodegenerative diseases and to determine the progression of MCI into AD.

\section{ACKNOWLEDGMENTS}

We thank Prof. S. Bastianello for supporting the MRI infrastructure in the initial phase of the work, Dr. Leda Roggeri for technical assistance, P. Chiarati and G. Germani for collaboration in data acquisition. The authors thank F. Negri, all the volunteers of the local recreational association "Argento Vivo" in Bereguardo, Pavia, Italy, and in particular their president T. Rampi for the enthusiastic to this study. This work was supported by grants of European Union to Egidio D'Angelo (Human Brain Project; HBP-604102) and of the Italian Ministry of Health to Egidio D'Angelo (RF-INM-2008-114341 and RF-2009-1475845). We also thank the Multiple Sclerosis International Federation (MSIF) for supporting the work with a Du Pre grant to Gloria Castellazzi.

\section{SUPPLEMENTARY MATERIAL}

The Supplementary Material for this article can be found online at: http://www.frontiersin.org/journal/10.3389/fnins.2014. 00223/abstract

\section{REFERENCES}

Agosta, F., Pievani, M., Geroldi, C., Copetti, M., Frisoni, G. B., and Filippi, M. (2012). Resting state fMRI in Alzheimer's disease: beyond the default mode network. Neurobiol. Aging 33, 1564-1578. doi: 10.1016/j.neurobiolaging.2011.06.007

Ashburner, J., and Friston, K. J. (2001). Why voxel-based morphometry should be used. Neuroimage 14, 1238-1243. doi: 10.1006/nimg.2001.0961

Babiloni, C., Binetti, G., Cassetta, E., Cerboneschi, D., Dal Forno, G., Del Percio, C., et al. (2004). Mapping distributed sources of cortical rhythms in mild Alzheimer's disease. A multicentric EEG study. Neuroimage 22, 57-67. doi: 10.1016/j.neuroimage.2003.09.028

Babiloni, C., Vecchio, F., Altavilla, R., Tibuzzi, F., Lizio, R., Altamura, C., et al. (2014). Hypercapnia affects the functional coupling of resting state electroencephalographic rhythms and cerebral haemodynamics in healthy elderly subjects and in patients with amnestic mild cognitive impairment. Clin. Neurophysiol. 125, 685-693. doi: 10.1016/j.clinph.2013.10.002

Bai, F., Liao, W., Watson, D. R., Shi, Y., Wang, Y., Yue, C., et al. (2011a). Abnormal whole-brain functional connection in amnestic mild cognitive impairment patients. Behav. Brain Res. 216, 666-672. doi: 10.1016/j.bbr.2010.09.010

Bai, F., Liao, W., Watson, D. R., Shi, Y., Yuan, Y., Cohen, A. D., et al. (2011b). Mapping the altered patterns of cerebellar resting-state function in longitudinal amnestic mild cognitive impairment patients. J. Alzheimers. Dis. 23, 87-99. doi: 10.3233/JAD-2010-101533

Bai, F., Zhang, Z., Watson, D. R., Yu, H., Shi, Y., Yuan, Y., et al. (2009). Abnormal functional connectivity of hippocampus during episodic memory retrieval processing network in amnestic mild cognitive impairment. Biol. Psychiatry 65, 951-958. doi: 10.1016/j.biopsych.2008.10.017

Beckmann, C. F., Deluca, M., Devlin, J. T., and Smith, S. M. (2005). Investigations into resting-state connectivity using independent component analysis. Philos. Trans. R. Soc. Lond. B Biol. Sci. 360, 1001-1013. doi: 10.1098/rstb. 2005.1634

Binnewijzend, M. A., Schoonheim, M. M., Sanz-Arigita, E., Wink, A. M., Van Der Flier, W. M., Tolboom, N., et al. (2012). Resting-state fMRI changes in Alzheimer's disease and mild cognitive impairment. Neurobiol. Aging 33, 2018-2028. doi: 10.1016/j.neurobiolaging.2011.07.003

Biswal, B., Yetkin, F. Z., Haughton, V. M., and Hyde, J. S. (1995). Functional connectivity in the motor cortex of resting human brain using echo-planar MRI. Magn. Reson. Med. 34, 537-541. doi: 10.1002/mrm.1910340409

Blennow, K., De Leon, M. J., and Zetterberg, H. (2006). Alzheimer's disease. Lancet 368, 387-403. doi: 10.1016/S0140-6736(06)69113-7
Boccardi, M., Sabattoli, F., Laakso, M. P., Testa, C., Rossi, R., Beltramello, A., et al. (2005). Frontotemporal dementia as a neural system disease. Neurobiol. Aging 26, 37-44. doi: 10.1016/j.neurobiolaging.2004.02.019

Bonnelle, V., Ham, T. E., Leech, R., Kinnunen, K. M., Mehta, M. A., Greenwood, R. J., et al. (2012). Salience network integrity predicts default mode network function after traumatic brain injury. Proc. Natl. Acad. Sci. U.S.A. 109, 4690-4695. doi: 10.1073/pnas.1113455109

Borst, A., and Theunissen, F. E. (1999). Information theory and neural coding. Nat. Neurosci. 2, 947-957. doi: 10.1038/14731

Brier, M. R., Thomas, J. B., Snyder, A. Z., Benzinger, T. L., Zhang, D., Raichle, M. E., et al. (2012). Loss of intranetwork and internetwork resting state functional connections with Alzheimer's disease progression. J. Neurosci. 32, 8890-8899. doi: 10.1523/JNEUROSCI.5698-11.2012

Brodmann, K. (2006). Brodmann's Localisation in the Cerebral Cortex. The Principles of Comparative Localisation in the Cerebral Cortex Based on Cytoarchitectonics. New York, NY: Springer US.

Buckner, R. L., Snyder, A. Z., Shannon, B. J., Larossa, G., Sachs, R., Fotenos, A. F., et al. (2005). Molecular, structural, and functional characterization of Alzheimer's disease: evidence for a relationship between default activity, amyloid, and memory. J. Neurosci. 25, 7709-7717. doi: 10.1523/JNEUROSCI.217705.2005

Caffarra, P., Vezzadini, G., Dieci, F., Zonato, F., and Venneri, A. (2002). ReyOsterrieth complex figure: normative values in an Italian population sample. Neurol. Sci. 22, 443-447. doi: 10.1007/s100720200003

Calhoun, V. D., Adali, T., McGinty, V. B., Pekar, J. J., Watson, T. D., and Pearlson, G. D. (2001). fMRI activation in a visual-perception task: network of areas detected using the general linear model and independent components analysis. Neuroimage 14, 1080-1088. doi: 10.1006/nimg.2001.0921

Cole, D. M., Smith, S. M., and Beckmann, C. F. (2010). Advances and pitfalls in the analysis and interpretation of resting-state FMRI data. Front. Syst. Neurosci. 4:8. doi: $10.3389 /$ fnsys. 2010.00008

Cole, M. W., and Schneider, W. (2007). The cognitive control network: Integrated cortical regions with dissociable functions. Neuroimage 37, 343-360. doi: 10.1016/j.neuroimage.2007.03.071

Corbetta, M., Kincade, J. M., and Shulman, G. L. (2002). Neural systems for visual orienting and their relationships to spatial working memory. J. Cogn. Neurosci. 14, 508-523. doi: 10.1162/089892902317362029

Cordes, D., Haughton, V. M., Arfanakis, K., Carew, J. D., Turski, P. A., Moritz, C. H., et al. (2001). Frequencies contributing to functional connectivity in the cerebral cortex in "resting-state" data. AJNR Am. J. Neuroradiol. 22, 1326-1333.

Damoiseaux, J. S., Beckmann, C. F., Arigita, E. J., Barkhof, F., Scheltens, P., Stam, C. J., et al. (2008). Reduced resting-state brain activity in the "default network" in normal aging. Cereb. Cortex 18, 1856-1864. doi: 10.1093/cercor/bhm207

Damoiseaux, J. S., Rombouts, S. A., Barkhof, F., Scheltens, P., Stam, C. J., Smith, S. M., et al. (2006). Consistent resting-state networks across healthy subjects. Proc. Natl. Acad. Sci. U.S.A. 103, 13848-13853. doi: 10.1073/pnas.0601417103

D'Angelo, E., and Casali, S. (2012). Seeking a unified framework for cerebellar function and dysfunction: from circuit operations to cognition. Front. Neural Circuits 6:116. doi: 10.3389/fncir.2012.00116

Di, X., Biswal, B. B., and Initiative, A. S. D. N. (2012). Metabolic brain covariant networks as revealed by FDG-PET with reference to resting-state fMRI networks. Brain Connect. 2, 275-283. doi: 10.1089/brain.2012.0086

Diedrichsen, J., Balsters, J. H., Flavell, J., Cussans, E., and Ramnani, N. (2009). A probabilistic MR atlas of the human cerebellum. Neuroimage, 46, 39-46. doi: 10.1016/j.neuroimage.2009.01.045

Ferreira, L. K., Diniz, B. S., Forlenza, O. V., Busatto, G. F., and Zanetti, M. V. (2011). Neurostructural predictors of Alzheimer's disease: a meta-analysis of VBM studies. Neurobiol. Aging 32, 1733-1741. doi: 10.1016/j.neurobiolaging.2009.11.008

Filippini, N., Macintosh, B. J., Hough, M. G., Goodwin, G. M., Frisoni, G. B., Smith, S. M., et al. (2009). Distinct patterns of brain activity in young carriers of the APOE-epsilon4 allele. Proc. Natl. Acad. Sci. U.S.A. 106, 7209-7214. doi: 10.1073/pnas.0811879106

Filippini, N., Scassellati, C., Boccardi, M., Pievani, M., Testa, C., Bocchio-Chiavetto, L., et al. (2006). Influence of serotonin receptor 2A His452Tyr polymorphism on brain temporal structures: a volumetric MR study. Eur. J. Hum. Genet. 14, 443-449. doi: 10.1038/sj.ejhg. 5201573

Folstein, M. F., Folstein, S. E., and McHugh, P. R. (1975). "Mini-mental state". A practical method for grading the cognitive state of patients for the clinician. J. Psychiatr. Res. 12, 189-198. doi: 10.1016/0022-3956(75)90026-6 
Fox, M. D., and Raichle, M. E. (2007). Spontaneous fluctuations in brain activity observed with functional magnetic resonance imaging. Nat. Rev. Neurosci. 8, 700-711. doi: 10.1038/nrn2201

Frisoni, G. B., Testa, C., Sabattoli, F., Beltramello, A., Soininen, H., and Laakso, M. P. (2005). Structural correlates of early and late onset Alzheimer's disease: voxel based morphometric study. J. Neurol. Neurosurg. Psychiatr. 76, 112-114. doi: 10.1136/jnnp.2003.029876

Good, C. D., Scahill, R. I., Fox, N. C., Ashburner, J., Friston, K. J., Chan, D., et al. (2002). Automatic differentiation of anatomical patterns in the human brain: validation with studies of degenerative dementias. Neuroimage 17, 29-46. doi: 10.1006/nimg.2002.1202

Gour, N., Felician, O., Didic, M., Koric, L., Gueriot, C., Chanoine, V., et al. (2014). Functional connectivity changes differ in early and late-onset alzheimer's disease. Hum Brain Mapp. 35, 2978-2994. doi: 10.1002/hbm.22379

Greicius, M. D., Krasnow, B., Reiss, A. L., and Menon, V. (2003). Functional connectivity in the resting brain: a network analysis of the default mode hypothesis. Proc. Natl. Acad. Sci. U.S.A. 100, 253-258. doi: 10.1073/pnas.01350 58100

Greicius, M. D., Srivastava, G., Reiss, A. L., and Menon, V. (2004). Default-mode network activity distinguishes Alzheimer's disease from healthy aging: evidence from functional MRI. Proc. Natl. Acad. Sci. U.S.A. 101, 4637-4642. doi: 10.1073/pnas.0308627101

Habas, C., Kamdar, N., Nguyen, D., Prater, K., Beckmann, C. F., Menon, V., et al. (2009). Distinct cerebellar contributions to intrinsic connectivity networks. J. Neurosci. 29, 8586-8594. doi: 10.1523/JNEUROSCI.1868-09.2009

Hachinski, V. C., Iliff, L. D., Zilhka, E., Du Boulay, G. H., McAllister, V. L., Marshall, J., et al. (1975). Cerebral blood flow in dementia. Arch. Neurol. 32, 632-637. doi: 10.1001/archneur.1975.00490510088009

Herskovits, A. Z., Locascio, J. J., Peskind, E. R., Li, G., and Hyman, B. T. (2013). A Luminex assay detects amyloid $\beta$ oligomers in Alzheimer's disease cerebrospinal fluid. PLoS ONE 8:e67898. doi: 10.1371/journal.pone.0067898

Holtzman, D. M., Morris, J. C., and Goate, A. M. (2011). Alzheimer's disease: the challenge of the second century. Sci. Transl. Med. 3:77sr71. doi: 10.1126/scitranslmed.3002369

Houk, J. C., and Wise, S. P. (1995). Distributed modular architectures linking basal ganglia, cerebellum, and cerebral cortex: their role in planning and controlling action. Cereb. Cortex 5, 95-110. doi: 10.1093/cercor/5.2.95

Hyder, F., Fulbright, R. K., Shulman, R. G., and Rothman, D. L. (2013). Glutamatergic function in the resting awake human brain is supported by uniformly high oxidative energy. J. Cereb. Blood Flow Metab. 33, 339-347. doi: 10.1038/jcbfm.2012.207

Hyder, F., and Rothman, D. L. (2012). Quantitative fMRI and oxidative neuroenergetics. Neuroimage 62, 985-994. doi: 10.1016/j.neuroimage.2012.04.027

Janes, A. C., Nickerson, L. D., Frederick, B. E. B., and Kaufman, M. J. (2012). Prefrontal and limbic resting state brain network functional connectivity differs between nicotine-dependent smokers and non-smoking controls. Drug Alcohol Depend. 125, 252-259. doi: 10.1016/j.drugalcdep.2012.02.020

Jones, D. T., Machulda, M. M., Vemuri, P., McDade, E. M., Zeng, G., Senjem, M. L., et al. (2011). Age-related changes in the default mode network are more advanced in Alzheimer disease. Neurology 77, 1524-1531. doi: 10.1212/WNL.0b013e318233b33d

Kaufmann, L., Ischebeck, A., Weiss, E., Koppelstaetter, F., Siedentopf, C., Vogel, S. E., et al. (2008). An fMRI study of the numerical Stroop task in individuals with and without minimal cognitive impairment. Cortex 44, 1248-1255. doi: 10.1016/j.cortex.2007.11.009

Keren-Happuch, E., Chen, S. H., Ho, M. H., and Desmond, J. E. (2014). A metaanalysis of cerebellar contributions to higher cognition from PET and fMRI studies. Hum. Brain Mapp. 35, 593-615. doi: 10.1002/hbm.22194

Koch, W., Teipel, S., Mueller, S., Benninghoff, J., Wagner, M., Bokde, A. L., et al. (2012). Diagnostic power of default mode network resting state fMRI in the detection of Alzheimer's disease. Neurobiol. Aging 33, 466-478. doi: 10.1016/j.neurobiolaging.2010.04.013

Krienen, F. M., and Buckner, R. L. (2009). Segregated fronto-cerebellar circuits revealed by intrinsic functional connectivity. Cereb. Cortex 19, 2485-2497. doi: 10.1093/cercor/bhp135

La Joie, R., Perrotin, A., Barré, L., Hommet, C., Mézenge, F., Ibazizene, M., et al. (2012). Region-specific hierarchy between atrophy, hypometabolism, and $\beta$-amyloid $(\mathrm{A} \beta$ ) load in Alzheimer's disease dementia. J. Neurosci. 32, 16265-16273. doi: 10.1523/JNEUROSCI.2170-12.2012
McKhann, G. M., Knopman, D. S., Chertkow, H., Hyman, B. T., Jack, C. R., Kawas, C. H., et al. (2011). The diagnosis of dementia due to Alzheimer's disease: recommendations from the National Institute on Aging-Alzheimer's Association workgroups on diagnostic guidelines for Alzheimer's disease. Alzheimers Dement. 7, 263-269. doi: 10.1016/j.jalz.2011.03.005

Mechelli, A., Friston, K. J., Frackowiak, R. S., and Price, C. J. (2005). Structural covariance in the human cortex. J. Neurosci. 25, 8303-8310. doi: 10.1523/JNEUROSCI.0357-05.2005

Minati, L., Chan, D., Mastropasqua, C., Serra, L., Spanò, B., Marra, C., et al. (2014). Widespread alterations in functional brain network architecture in amnestic mild cognitive impairment. J. Alzheimers Dis. 40, 213-220. doi: 10.3233/JAD131766

Morbelli, S., Piccardo, A., Villavecchia, G., Dessi, B., Brugnolo, A., Piccini, A., et al. (2010). Mapping brain morphological and functional conversion patterns in amnestic MCI: a voxel-based MRI and FDG-PET study. Eur. J. Nucl. Med. Mol. Imaging 37, 36-45. doi: 10.1007/s00259-009-1218-6

Mormino, E. C., Smiljic, A., Hayenga, A. O., Onami, S. H., Greicius, M. D., Rabinovici, G. D., et al. (2011). Relationships between $\beta$-amyloid and functional connectivity in different components of the default mode network in aging. Cereb. Cortex 21, 2399-2407. doi: 10.1093/cercor/bhr025

Ni, R., Gillberg, P. G., Bergfors, A., Marutle, A., and Nordberg, A. (2013). Amyloid tracers detect multiple binding sites in Alzheimer's disease brain tissue. Brain 136, 2217-2227. doi: 10.1093/brain/awt142

Novelli, G., Papagno, C., Capitani, E., Laiacona, M., Cappa, S. F., and Vallar, G. (1986). Three clinical tests for the assessment of verbal long-term memory function: Norms from 320 normal subjects. Arch. Psicol. Neurol. Psichiatr. 47, 477-506.

Oakes, T. R., Fox, A. S., Johnstone, T., Chung, M. K., Kalin, N., and Davidson, R. J. (2007). Integrating VBM into the General Linear Model with voxelwise anatomical covariates. Neuroimage 34, 500-508. doi: 10.1016/j.neuroimage.2006.10.007

Osterrieth, P. A. (1944). Le test de copie d'une figure complex: Contribution à l'étude de la perception et de la memoir. Arch. Psychol. 30, 286-356.

Pennanen, C., Testa, C., Laakso, M. P., Hallikainen, M., Helkala, E. L., Hänninen, T., et al. (2005). A voxel based morphometry study on mild cognitive impairment. J. Neurol. Neurosurg. Psychiatr. 76, 11-14. doi: 10.1136/jnnp.2004. 035600

Petersen, R. C. (2009). Early diagnosis of Alzheimer's disease: is MCI too late? Curr. Alzheimer Res. 6, 324-330. doi: 10.2174/156720509788929237

Petersen, R. C., Roberts, R. O., Knopman, D. S., Boeve, B. F., Geda, Y. E., Ivnik, R. J., et al. (2009). Mild cognitive impairment: ten years later. Arch. Neurol. 66, 1447-1455. doi: 10.1001/archneurol.2009.266

Petersen, R. C., Stevens, J. C., Ganguli, M., Tangalos, E. G., Cummings, J. L., and Dekosky, S. T. (2001). Practice parameter: early detection of dementia: mild cognitive impairment (an evidence-based review). Report of the quality standards subcommittee of the american academy of neurology. Neurology 56, 1133-1142. doi: 10.1212/WNL.56.9.1133

Petrella, J. R., Sheldon, F. C., Prince, S. E., Calhoun, V. D., and Doraiswamy, P. M. (2011). Default mode network connectivity in stable vs progressive mild cognitive impairment. Neurology 76, 511-517. doi: 10.1212/WNL.0b013e31820af94e

Raichle, M. E., Macleod, A. M., Snyder, A. Z., Powers, W. J., Gusnard, D. A., and Shulman, G. L. (2001). A default mode of brain function. Proc. Natl. Acad. Sci. U.S.A. 98, 676-682. doi: 10.1073/pnas.98.2.676

Randolph, C., Braun, A. R., Goldberg, T. E., and Chase, T. N. (1993). Semantic fluency in Alzheimer's, Parkinson's, and Huntington's disease: dissociation of storage and retrieval failures. Neuropsychology 1, 82-88. doi: 10.1037/08944105.7.1.82

Reitan, R. M. (1958). Validity of the Trail making test as an indicator of organic brain damage. Percept. Mot. Skills 8, 271-276. doi: 10.2466/pms.1958.8.3.271

Ries, M. L., Carlsson, C. M., Rowley, H. A., Sager, M. A., Gleason, C. E., Asthana, S., et al. (2008). Magnetic resonance imaging characterization of brain structure and function in mild cognitive impairment: a review. J. Am. Geriatr. Soc. 56, 920-934. doi: 10.1111/j.1532-5415.2008.01684.x

Schmahmann, J. D., Doyon, J., McDonald, D., Holmes, C., Lavoie, K., Hurwitz, A. S., et al. (1999). Three-dimensional MRI atlas of the human cerebellum in proportional stereotaxic space. Neuroimage 10, 233-260. doi: 10.1006/nimg.1999.0459

Seeley, W. W., Crawford, R. K., Zhou, J., Miller, B. L., and Greicius, M. D. (2009). Neurodegenerative diseases target large-scale human brain networks. Neuron 62, 42-52. doi: 10.1016/j.neuron.2009.03.024 
Serrano-Pozo, A., Frosch, M. P., Masliah, E., and Hyman, B. T. (2011). Neuropathological alterations in Alzheimer disease. Cold Spring Harb. Perspect. Med. 1:a006189. doi: 10.1101/cshperspect.a006189

Sluimer, J. D., Van Der Flier, W. M., Karas, G. B., Van Schijndel, R., Barnes, J., Boyes, R. G., et al. (2009). Accelerating regional atrophy rates in the progression from normal aging to Alzheimer's disease. Eur. Radiol. 19, 2826-2833. doi: 10.1007/s00330-009-1512-5

Smith, S. M., Fox, P. T., Miller, K. L., Glahn, D. C., Fox, P. M., Mackay, C. E., et al. (2009). Correspondence of the brain's functional architecture during activation and rest. Proc. Natl. Acad. Sci. U.S.A. 106, 13040-13045. doi: 10.1073/pnas.0905 267106

Smith, S. M., and Nichols, T. E. (2009). Threshold-free cluster enhancement: addressing problems of smoothing, threshold dependence and localisation in cluster inference. Neuroimage 44, 83-98. doi: 10.1016/j.neuroimage.2008. 03.061

Solé-Padullés, C., Bartrés-Faz, D., Junqué, C., Vendrell, P., Rami, L., Clemente, I. C., et al. (2009). Brain structure and function related to cognitive reserve variables in normal aging, mild cognitive impairment and Alzheimer's disease. Neurobiol. Aging 30, 1114-1124. doi: 10.1016/j.neurobiolaging.2007.10.008

Spinnler, H., and Dall'ora, P. (1987). On Alzheimer's disease: an overview of diagnostic and research issues for the clinical neurologist. Funct. Neurol. 2, 5-36.

Spinnler, H., and Tognoni, G. (1987). Standardizzazione e taratura italiana di test neuropsicologici. Ital. J. Neurol. Sci. 6, 8-72.

Spreng, R. N., Mar, R. A., and Kim, A. S. (2009). The common neural basis of autobiographical memory, prospection, navigation, theory of mind, and the default mode: a quantitative meta-analysis. J. Cogn. Neurosci. 21, 489-510. doi: 10.1162/jocn.2008.21029

Stoodley, C. J. (2012). The cerebellum and cognition: evidence from functional imaging studies. Cerebellum 11, 352-365. doi: 10.1007/s12311-011-0260-7

Stoodley, C. J., and Schmahmann, J. D. (2010). Evidence for topographic organization in the cerebellum of motor control versus cognitive and affective processing. Cortex 46, 831-844. doi: 10.1016/j.cortex.2009.11.008

Stoodley, C. J., Valera, E. M., and Schmahmann, J. D. (2012). Functional topography of the cerebellum for motor and cognitive tasks: an fMRI study. Neuroimage 59, 1560-1570. doi: 10.1016/j.neuroimage.2011.08.065

Teipel, S. J., Grothe, M., Lista, S., Toschi, N., Garaci, F. G., and Hampel, H. (2013). Relevance of magnetic resonance imaging for early detection and diagnosis of Alzheimer disease. Med. Clin. North Am. 97, 399-424. doi: 10.1016/j.mcna.2012.12.013

Teipel, S. J., Meindl, T., Grinberg, L., Heinsen, H., and Hampel, H. (2008). Novel MRI techniques in the assessment of dementia. Eur. J. Nucl. Med. Mol. Imaging 35(Suppl. 1), S58-S69. doi: 10.1007/s00259-007-0703-Z

Testa, C., Laakso, M. P., Sabattoli, F., Rossi, R., Beltramello, A., Soininen, H., et al. (2004). A comparison between the accuracy of voxel-based morphometry and hippocampal volumetry in Alzheimer's disease. J. Magn. Reson. Imaging 19, 274-282. doi: 10.1002/jmri.20001
Thomann, P. A., Schläfer, C., Seidl, U., Santos, V. D., Essig, M., and Schröder, J. (2008). The cerebellum in mild cognitive impairment and Alzheimer's disease - a structural MRI study. J. Psychiatr. Res. 42, 1198-1202. doi: 10.1016/j.jpsychires.2007.12.002

Tomasi, D., Wang, G. J., and Volkow, N. D. (2013). Energetic cost of brain functional connectivity. Proc. Natl. Acad. Sci. U.S.A. 110, 13642-13647. doi: 10.1073/pnas.1303346110

Wang, K., Liang, M., Wang, L., Tian, L., Zhang, X., Li, K., et al. (2007). Altered functional connectivity in early Alzheimer's disease: a resting-state fMRI study. Hum. Brain Mapp. 28, 967-978. doi: 10.1002/hbm.20324

Wang, L., Zang, Y., He, Y., Liang, M., Zhang, X., Tian, L., et al. (2006) Changes in hippocampal connectivity in the early stages of Alzheimer's disease: evidence from resting state fMRI. Neuroimage 31, 496-504. doi: 10.1016/j.neuroimage.2005.12.033

Wu, C. W., Gu, H., Lu, H., Stein, E. A., Chen, J. H., and Yang, Y. (2009). Mapping functional connectivity based on synchronized $\mathrm{CMRO} 2$ fluctuations during the resting state. Neuroimage 45, 694-701. doi: 10.1016/j.neuroimage.2008.12.066

Wu, X., Li, R., Fleisher, A. S., Reiman, E. M., Guan, X., Zhang, Y., et al. (2011). Altered default mode network connectivity in Alzheimer's disease-a resting functional MRI and Bayesian network study. Hum. Brain Mapp. 32, 1868-1881. doi: $10.1002 / \mathrm{hbm} .21153$

Zhang, D., and Raichle, M. E. (2010). Disease and the brain's dark energy. Nat. Rev. Neurol. 6, 15-28. doi: 10.1038/nrneurol.2009.198

Zhang, H. Y., Wang, S. J., Xing, J., Liu, B., Ma, Z. L., Yang, M., et al. (2009). Detection of PCC functional connectivity characteristics in resting-state fMRI in mild Alzheimer's disease. Behav. Brain Res. 197, 103-108. doi: 10.1016/j.bbr.2008.08.012

Conflict of Interest Statement: The authors declare that the research was conducted in the absence of any commercial or financial relationships that could be construed as a potential conflict of interest.

Received: 13 March 2014; accepted: 07 July 2014; published online: 30 July 2014. Citation: Castellazzi G, Palesi F, Casali S, Vitali P, Sinforiani E, Wheeler-Kingshott CAM and D'Angelo E (2014) A comprehensive assessment of resting state networks: bidirectional modification of functional integrity in cerebro-cerebellar networks in dementia. Front. Neurosci. 8:223. doi: 10.3389/fnins.2014.00223

This article was submitted to Brain Imaging Methods, a section of the journal Frontiers in Neuroscience.

Copyright (C) 2014 Castellazzi, Palesi, Casali, Vitali, Sinforiani, Wheeler-Kingshott and D'Angelo. This is an open-access article distributed under the terms of the Creative Commons Attribution License (CC BY). The use, distribution or reproduction in other forums is permitted, provided the original author(s) or licensor are credited and that the original publication in this journal is cited, in accordance with accepted academic practice. No use, distribution or reproduction is permitted which does not comply with these terms. 